\title{
Optimal Energy Management for Formula-E Cars with Regulatory Limits and Thermal Constraints
}

\author{
Xuze Liu, Abbas Fotouhi*, Daniel J. Auger \\ Advanced Vehicle Engineering Centre, School of Aerospace, Transport and Manufacturing (SATM) \\ Cranfield University, Cranfield, Bedfordshire, MK43 0AL, UK \\ *Corresponding Author, email: a.fotouhi@cranfield.ac.uk
}

\begin{abstract}
In this paper, novel solutions are proposed for energy and thermal management in Formula-E cars using optimal control theory. Optimal control techniques are used to optimize net energy consumption (accounting for loss-reductions from energy recovery from regenerative braking) to achieve minimal lap time which is a crucial element in developing a competitive race strategy in Formula E races. A thermal battery model is used to impose thermal constraints on the optimal energy management strategy in order to realistically capture working constraints during a race. The effects of energy and thermal constraints on the proposed strategy are then demonstrated and two different pedal lifting techniques were introduced. Both the current second generation and a concept third generation type of formula-E cars are studied and compared. While third generation is significantly more efficient with $10 \%$ to $30 \%$ less energy consumption, it potentially faces more critical thermal issues with more than $60 \%$ more heat generation.
\end{abstract}

Key words: Formula-E car; optimal control; lap time simulation; energy management; thermal management

\section{Introduction}

Energy management has been a popular topic in vehicles with multiple energy sources (e.g. fuel and battery [1]) or multiple motors(e.g. dual axle drive [2]). A range of strategies and algorithms have been proposed in the literature to manage the power flow among the sources and consumers such as rule-based fuzzy control [3] and optimization-based control [4]. Such techniques are applied in real-time and usually tested on widely-used driving cycles to verify their effectiveness in improving energy economy. Apart from real-time applications, a number of studies took the entire driving cycle or route as a fixed known condition in the trip-based optimizations aiming to solve a global optimal solution for the entire trip [5] given boundaries such as battery state of charge (SOC) or time[6]. In the modern motorsport, energy management is a crucial problem in race events. To create a fair competition environment, the governing bodies' technical regulations have imposed upper limits on power and energy. For example, for the hybrid system in current Formula-One cars, the technical regulations state that fuel mass flow rate for the internal combustion engine (ICE) must not exceed $100 \mathrm{~kg} / \mathrm{h}$ and the maximum power for the Motor Generator Unit - Kinetic (MGUK) is limited to $120 \mathrm{~kW}$ [7]. In Formula-E, this appears as restrictions on the output power out of a car's Rechargeable Energy Storage System (RESS), which may vary for different events (e.g. qualifying, race, etc.) and restrictions on the amount of energy that can be delivered to the Motor Generator Unit (MGU) [8]. With these restrictions introduced, drivers and engineers are not able to go 'flat 
out' during the race simply because this is not efficient and there is not enough energy on board. Therefore, managing 'energy per lap' under various constraints while optimizing the lap times has become a crucial part in developing a competitive race strategy in Formula-E races.

Simulation and optimization have been the core of motorsport performance and strategy analysis. Driver-in-the-loop (DIL) simulation is a high-level real-time simulation method with high-fidelity models for validation and assessment. While this method is capable of involving human element in the simulation and provides detailed and direct feedback from the virtual environment for sophisticated tasks [9], it is very slow in terms of optimization iterations as simulations must be performed in real time and outside these simulation tools due to the lack of embedded powerful optimization algorithms which is the common weakness shared by commercial software. Therefore traditionally, these high-fidelity tools are usually used as a validation tool to test a strategy which is established through optimizations before hand. A classic development procedure can be demonstrated with figure 1 . This study focuses on step 1 and 2 which lays the foundation for highfidelity validations. This requires techniques with high computational efficiency and optimization capabilities for lap time analysis.

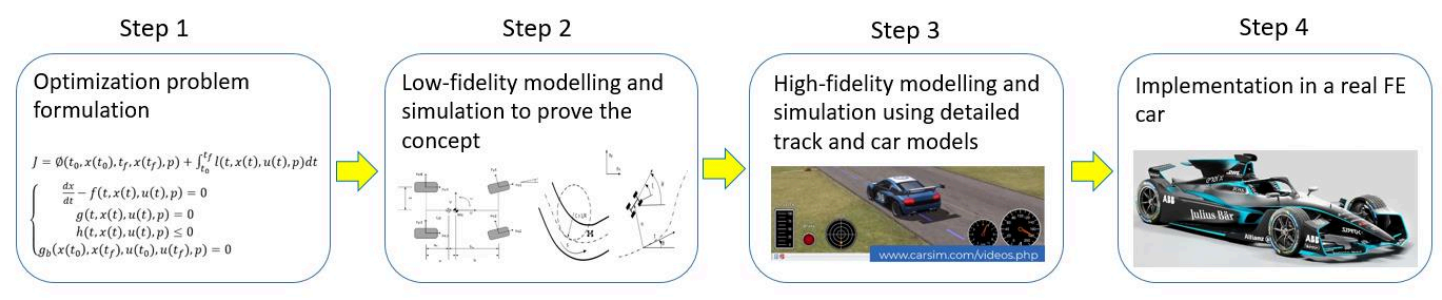

Figure 1: Classic development steps of FE race strategy optimization, modelling and implementation

In the early 2000s, quasi-steady-state (QSS) methods were proposed and have become a popular method for performance sensitivity analysis [10]. The introduction of QSS methods has saved tremendous time that would have otherwise been spent testing different configurations either on track or DIL simulators. In these methods, as its label 'quasi-steady-state' suggests, the vehicle models are commonly rather simplified; optimization horizons are often short(meters level) so these methods have less utility when a cost function need to be minimized subject to a series of constraints being met through the whole lap. Model predictive control (MPC), in contrary, is capable to accommodate more complex models and various constraints. However, MPCs are most powerful being applied as a real-time controller [11] based on iterative, finite-horizon optimization. Note that the energy and thermal problem in Formula $\mathrm{E}$ is a management problem whose optimization horizon should be the length of a whole lap. This fundamental feature makes MPC unfavourable because of its shorter optimization horizon may easily yield a suboptimal solution.

Another approach is to formulate an optimal control problem (OCP). By taking driver inputs (Throttle, brake, etc.) as a sequence of inputs and applying constraints such as track boundaries, calculation for minimal time maneuvers (MTM) is an typical example of OCP. Early OCPs with smaller multibody dynamics model such as a motorbike [12] or track as simple as a U-turn [13] were solved with indirect methods based on Pontryagin Maximum Principle [14]. In recent years, direct methods based on full collocation have become more popular, thanks to the solver IPOPT [15] that is an efficient interior point algorithm for solving large nonlinear programming (LNP) problem resulted from direct collocation. The computational time of a full-lap simulation has been reduced 
from dozens of hours [16] using a direct multiple shooting technique to acceptable minute-level [17].

A number of recent publications in the literature have been focused on different methods of track and vehicle modeling. Giacomo proposed modeling the track using a curvilinear-coordinate-based description of the centre line in both 2D [18] and 3D [19] ways. The track was generated by solving an OCP based on GPS data to reduce the noise level in the coordinates. The complexity of vehicle modeling has a big effect on computational time and solubility of the optimization problem. Recent literatures adopt different simplified modelling methods targeting different problems. For example, differential optimization has been studied in [18] Where the slip rate of four wheels were taken as optimal control inputs. A limited-slip differential was modeled and applied on rear axle as a path constraint for the problem. But involving front wheels in the inputs allows traction to the front wheels which is not realistic and by directly propelling each wheel with differential constraint may result in solution that is not doable in real life. In [20], drive torque was set as optimal input with wheels/tyres as additional degree of freedoms (DOFs). The front wheels are free-rolling with drags adding wheel rotation DOFs. The differential torque was calculated based on rear wheel speed differential then added on each rear wheel dynamic equations. But this study was only completed on a U-shape turn. Tyre is one of the most crucial elements in motorsport vehicles. A thermaldynamic tyre model was introduced in [21] to study the influence of tyre, track and ambient temperature on the MTM. Tremlett [22] added temperature-friction sensitivity and wear model into the OCP in which the optimal tyre warming and usage strategy were studied. Apart from the tyres, based on the work in [18], Limebeer[23] modelled the energy recovery systems (ERSs) and demonstrated that a third of fuel can be saved by using hybrid power system in a Formula One car. Masouleh [24] added quasi-static aero-suspension model in the OCP to study the optimization of aero-suspension interaction.

In Formula E championships season 2020, the technical regulation [8] restricts the use of energy in various ways. In term of power out of the RESS, in qualifications where drivers compete for their starting position for the race start, the limit is set to $250 \mathrm{~kW}$ whereas in race events, $200 \mathrm{~kW}$ and $235 \mathrm{~kW}$ are set as limits for normal and attack modes respectively. For the energy restrictions, a driver who has a Fanboost bonus will be given an extra energy of $100 \mathrm{~kJ}$ to be used while raising the max power from $200 \mathrm{~kW}$ in normal mode by $40-50 \mathrm{~kW}$. However, the total amount of energy that can be delivered to the MGU during a race still must not exceed $52 \mathrm{kWh}$. In addition to the energy restrictions, battery thermal dynamics is another crucial factor to concern in strategy development. Because of safety reasons, the battery only functions normally under a certain temperature limit. After reaching the limit, the battery management system (BMS) will automatically shut down the power. As a result, a driver with an overheating battery may lose his positions or even have a Did-Not-Finish (DNF) for the race.

According to the literature and to the authors' knowledge and experience, applying optimal control methods to solve the energy/thermal management problem of a Formula-E car has the following advantages:

1. It is capable to optimize the control inputs and race car trajectory simultaneously. The latter contributes to a unique feature in formula-E compared to other series because of the attack mode when the drivers need to drive through an activation zone that is deviated from normal race lines.

2. It is powerful enough to deal with complex models, e.g. higher vehicle DOFs, non-linear 
tyre models, thermal models, etc.;

3. It can deal with non-fixed constraints on different sections of the track, e.g. change of power restrictions due to different power mode or yellow flag scenarios; it can be formulated into a multi-phase optimal control problem.

4. It is a robust method requiring less from initial guess and cost decent amount of computational time (dozens of minutes)

In this paper, orthogonal collocation method [25] is used to solve the optimal control problem of Formula-E energy/thermal management. Recent seasons from 2018 have been featured with second generation Formula-E cars with upgraded battery and other features which significantly improved the performance in comparison to the first generation. In December 2019, Fédération Internationale de l'Automobile (FIA; English: International Automobile Federation) published the tenders for the third generation of formula $\mathrm{E}$ race cars which will be used from 2022 onwards. This allows the public to have a preview of potential specifications of future electric race cars.

Table 1: Specifications of different generations of Formula-E cars

\begin{tabular}{|l|l|l|l|l|}
\hline Generation & Weight & Battery capacity & Drive type & Max power \\
\hline Gen1 & $880 \mathrm{~kg}$ & $28 \mathrm{kWh}$ & Rear wheel drive & $200 \mathrm{~kW}$ \\
\hline Gen2 & $900 \mathrm{~kg}$ & $52 \mathrm{kWh}$ & Rear wheel drive & $250 \mathrm{~kW}$ \\
\hline $\begin{array}{l}\text { Gen3 } \\
\text { (Tender option A) }\end{array}$ & $780 \mathrm{~kg}$ & $51 \mathrm{kWh}$ & $\begin{array}{l}\text { Rear wheel drive } \\
\text { Front \& rear axle } \\
\text { regeneration }\end{array}$ & $350 \mathrm{~kW}$ \\
\hline
\end{tabular}

The most radical change is the powertrain configuration. The current Gen 2 cars are propelled by the MGU through the rear axle. From season 2022, the Gen3 cars will feature one MGU on each axle, propelled only by the rear one and regenerate energy from both front and rear MGUs which indicates that more energy will be recovered from front axle through braking. This study is focused on the energy/thermal management of the Gen 2 cars then a prospective study on the succeeding Gen 3 cars. In Section 2, track model is introduced. The vehicle is modelled next with non-linear tyre models and other essential DOFs to describe the features of both Gen2 and Gen3 cars. Then a thermal dynamic model of the powertrain system is presented which includes a lump thermal model of the battery and the heat exchange model for the liquid cooling system. In Section 3, orthogonal collocation method for optimal control technique is briefly explained and the energy/thermal management problem is formulated for OCP. The result and discussion of energy/thermal management for Gen2 cars under different constraints and environment conditions are presented in Section 4 along with the perspective study of Gen3 cars and their comparison. Finally, the conclusion and potential future works are presented in Section 5.

The novelty of this study is that 1) optimal control techniques are used to study the optimal management solution under both energy and thermal constraints; 2) two different powertrain configurations are investigated; 3) a novel technique to reduce battery temperature rise has been found. 


\section{Track, vehicle and thermal dynamics model}

\subsection{Track model}

In this paper, the track is modeled using curvilinear coordinates instead of traditional xy coordinates. The curvature of a point on the track and the centerline distance from the start/finish line of that point are treated as ordinate and abscissa values.

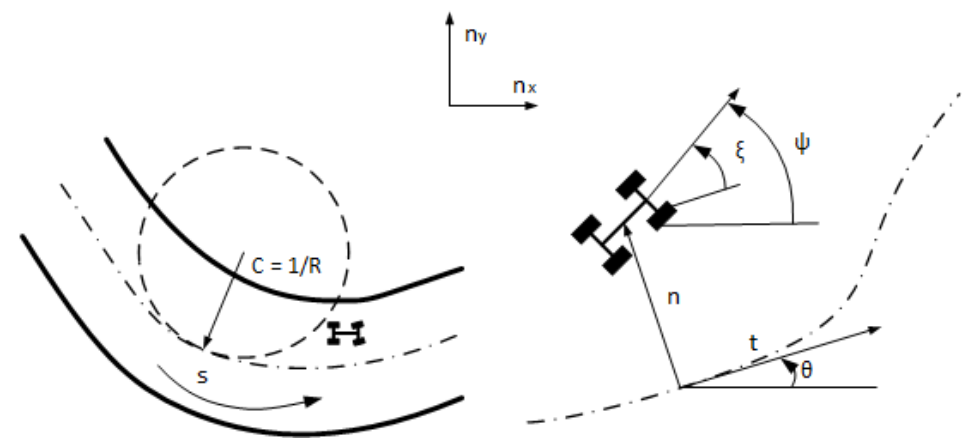

Figure 2: Track modelling coordinate

Illustrated in figure 2, the location of the vehicle on the track can be described using $\mathrm{s}$ and $\mathrm{n}$, of which the former gives the distance traveled along the centerline from the start/finish line and the latter gives the perpendicular distance of the vehicle mass center from the centerline to the centerline's tangent direction.

Followed by Perantoni [18], the lateral position $\mathrm{n}$ is calculated using its derivative:

$$
\dot{n}=u \sin \xi+v \cos \xi
$$

In which $\mathrm{u}$ and $\mathrm{v}$ represent the longitudinal and lateral velocity of the vehicle respectively. The parameter $\xi$ is the angle between the vehicle heading direction and the track centerline tangent direction and is calculated by:

$$
\dot{\xi}=\dot{\psi}-C \dot{s}
$$

The traveled distance $\mathrm{s}$ can therefore be calculated by

$$
\dot{s}=\frac{u \cos \xi-v \sin \xi}{1-n C}
$$

C gives the centerline curvature of the abscissa point where the vehicle is located. For a left turn, $\mathrm{C}$ is positive and $\mathrm{n}$ is positive when the vehicle is on the left hand side of the centerline. It should be noted that the dot notation in this paper denote that these are derivatives with respect to time. The traveled distance $\mathrm{s}$ is a monotonically increasing function of time because cars are not supposed to go backwards on the track realistically. In order to minimize the lap time $t$ in the OCP, $s$ is used as an independent variable. According to equation (4), the derivatives respective to time can be converted as respective to distance by multiplying the time derivatives by $S_{f}$ where the $S_{f}$ is the reciprocal of velocity of vehicle along the track centerline.

$$
\begin{aligned}
& d t=\frac{d t}{d s} d s=\frac{1-n C}{u \cos \xi-v \sin \xi} d s \\
& S_{f}=\left(\frac{d s}{d t}\right)^{-1}=\frac{1-n C}{u \cos \xi-v \sin \xi}
\end{aligned}
$$

There follows 


$$
\frac{d n}{d s}=(u \sin \xi+v \cos \xi) S_{f}
$$

and

$$
\frac{d \xi}{d s}=S_{f} \omega-C
$$

where $\omega=\dot{\psi}$ is the vehicle yaw rate.

\subsection{Vehicle model}

We choose not to use higher-fidelity models to include pitch and roll motions in this study because the motions of pitch and roll are more of a priority when a task is more dominated by vehicle dynamics studies. In high level motorsport (Formula one, Formula E), the roll and pitch motions themselves have relatively low magnitudes due to the high stiffness of suspensions on these cars. The main reason why these are important is that they have crucial effect on aerodynamics performance like in Formula One where aerodynamics downforce character is one of the dominant factor thus cannot be overlooked in performance analysis[24] . However in Formula E, the body work used by teams are identical and its design concept is very different from formula one [26] and is of much less concern in downforce generation thus the performance is less aerodynamic dependent or sensitive to pitch and roll motions[27] . Therefore, the vehicle used in this paper is modeled with 7 DOFs including longitudinal, lateral, yaw motions and 4 wheel rotations with aerodynamic loads and load transfer taken into account. A limited-slip differential (LSD) is modelled due to the fact that the cars are driven by one motor per axle.

\subsubsection{Body dynamics}

As illustrated in figure 3, the longitudinal, lateral and yaw motions are described using the following equations:

$$
\begin{gathered}
M \frac{d}{d t} u(t)=M \omega v+F_{x} \\
M \frac{d}{d t} v(t)=-M \omega u+F_{y} \\
I_{z} \frac{d}{d t} \omega(t)=a\left(\cos \delta\left(F_{y 1}+F_{y 2}\right)+\sin \delta\left(F_{x 1}+F_{x 2}\right)\right)-w\left(\sin \delta F_{y 2}-\cos \delta F_{x 2}\right)+w F_{x 3}- \\
w\left(\cos \delta F_{x 1}-\sin \delta F_{y 1}\right)-w F_{x 1}-b\left(F_{y 3}+F_{y 4}\right)
\end{gathered}
$$

where $M$ is the vehicle mass, $I_{z}$ is the vehicle yaw moment of inertia, $\delta$ is the front wheel steering angle and $w$ is the track width. $a$ and $b$ are the distances from the vehicle's center of mass to the front and reat axle respectively. The $F_{x i}$ and $F_{y i}$ are the longitudinal and lateral forces respectively generated by the tyres with subscript i $(i=1,2,3,4)$ denoting the tyre on the front left, front right, rear right and rear left. The total longitudinal and lateral forces $F_{x}$ and $F_{y}$ acting on vehicle are given by

$$
\begin{gathered}
F_{x}=\cos \delta\left(F_{x 1}+F_{x 2}\right)-\sin \delta\left(F_{y 1}+F_{y 2}\right)+\left(F_{x 3}+F_{x 4}\right)-F_{a x} \\
F_{y}=\cos \delta\left(F_{y 1}+F_{y 2}\right)+\sin \delta\left(F_{x 1}+F_{x 2}\right)+\left(F_{y 3}+F_{y 4}\right)
\end{gathered}
$$

where $F_{a x}$ is the longitudinal aerodynamic load on the car which will be defined in Section 2.2.3. 


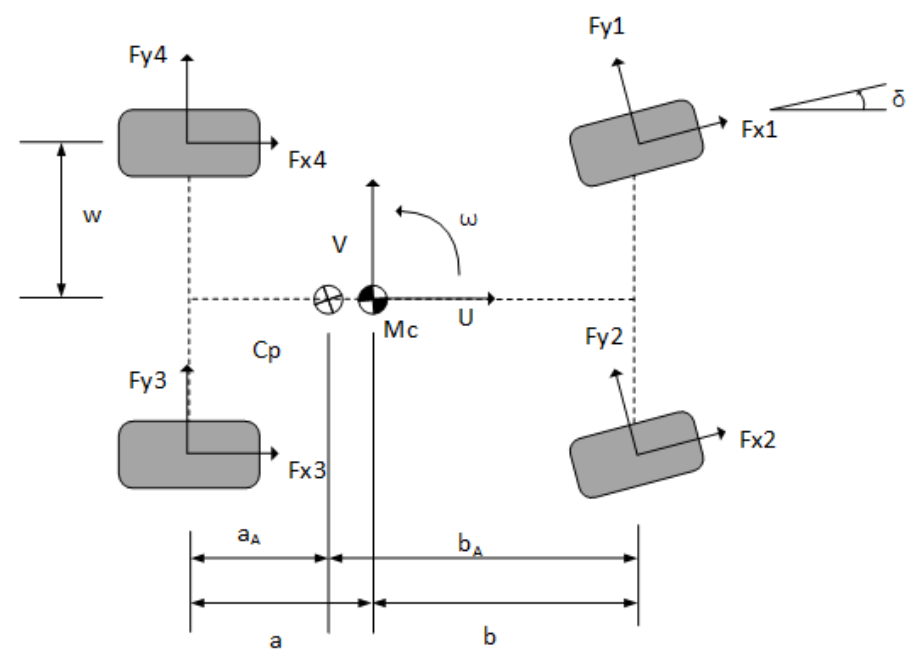

Figure 3: 7-DOF Vehicle model

\subsubsection{Wheel dynamics}

The tyre model used in this paper is an empirical tyre model [28] with linearized interpolation for peak values of longitudinal and lateral friction coefficient [29]. The longitudinal and lateral forces are given by:

$$
\begin{aligned}
& F_{x i}=\mu_{x i} F_{z i} \frac{\kappa_{n i}}{\sqrt{\alpha_{n i}^{2}+\kappa_{n i}^{2}+\epsilon}} \\
& F_{y i}=\mu_{y i} F_{z i} \frac{\alpha_{n i}}{\sqrt{\alpha_{n i}^{2}+\kappa_{n i}^{2}+\epsilon}}
\end{aligned}
$$

where $F_{z i}$ is the tyre normal force, $\kappa_{n i}$ and $\alpha_{n i}$ are the normalized tyre slip with respect to the slip value where peak friction coefficient happens:

$$
\begin{aligned}
& \alpha_{n i}=\frac{\alpha_{i}}{\alpha_{\max i}} \\
& \kappa_{n i}=\frac{\kappa_{i}}{\kappa_{\max i}}
\end{aligned}
$$

The slip angles $\alpha_{n i}$ and ratios $\kappa_{n i}$ are given by:

$$
\begin{gathered}
\alpha_{1}=\delta-\arctan \left(\frac{v+\omega a}{u-\omega w}\right), \kappa_{1}=-1+\frac{R \omega_{1}}{\cos \delta(u-\omega w)-\sin \delta(v+\omega a)} \\
\alpha_{2}=\delta-\arctan \left(\frac{v+\omega a}{u+\omega w}\right), \kappa_{2}=-1+\frac{R \omega_{2}}{\cos \delta(u+\omega w)-\sin \delta(v+\omega a)} \\
\alpha_{3}=-\arctan \left(\frac{v-\omega b}{u+\omega w}\right), \quad \kappa_{3}=-1+\frac{R \omega_{3}}{u+\omega w} \\
\alpha_{4}=-\arctan \left(\frac{v-\omega b}{u-\omega w}\right), \kappa_{4}=-1+\frac{R \omega_{4}}{u-\omega w}
\end{gathered}
$$

In which $\omega_{i}$ is the angular velocity of each wheel and $\mathrm{R}$ the tyre radius.

The peak friction values are given by linear interpolation which treats them as linear function or the tyre normal loads. The symbols are explained in table2.

$$
\mu_{x \max }=\left(F_{Z}-F_{Z R 1}\right)\left(\frac{\mu_{x \max 2}-\mu_{x \max 1}}{F_{Z R 2}-F_{Z R 1}}\right)+\mu_{x \max 1}
$$




$$
\begin{gathered}
\mu_{y \max }=\left(F_{z}-F_{z R 1}\right)\left(\frac{\mu_{y \max 2}-\mu_{y \max 1}}{F_{Z R 2}-F_{z R 1}}\right)+\mu_{y \max 1} \\
\kappa_{\max }=\left(F_{z}-F_{Z R 1}\right)\left(\frac{\kappa_{\max 2}-\kappa_{\max 1}}{F_{Z R 2}-F_{Z R 1}}\right)+\kappa_{\max 1} \\
\alpha_{\max }=\left(F_{Z}-F_{Z R 1}\right)\left(\frac{\alpha_{\max 2}-\alpha_{\max 1}}{F_{Z R 2}-F_{Z R 1}}\right)+\alpha_{\max 1}
\end{gathered}
$$

Table 2 Description of symbols

\begin{tabular}{|c|l|l|l|}
\hline Symbol & Description & Front value & Rear value \\
\hline$F_{Z R 1}$ & Reference normal load 1 & $2000 \mathrm{~N}$ & $2000 \mathrm{~N}$ \\
\hline$F_{z R 2}$ & Reference normal load 2 & $6000 \mathrm{~N}$ & $6000 \mathrm{~N}$ \\
\hline$\mu_{x \max 1}$ & Peak longitudinal friction coefficient at load 1 & 1.4 & 1.75 \\
\hline$\mu_{x \max 2}$ & Peak longitudinal friction coefficient at load 2 & 1.12 & 1.4 \\
\hline$\kappa_{\max 1}$ & Slip coefficient for the friction peak at load 1 & 0.11 & 0.11 \\
\hline$\kappa_{\max 2}$ & Slip coefficient for the friction peak at load 2 & 0.10 & 0.10 \\
\hline$\mu_{y \max 1}$ & Peak lateral friction coefficient at load 1 & 1.62 & 1.8 \\
\hline$\mu_{y} \max 2$ & Peak lateral friction coefficient at load 2 & 1.3 & 1.45 \\
\hline$\alpha_{\max 1}$ & Slip angle for the friction peak at load 1 & $9^{\circ}$ & $9^{\circ}$ \\
\hline$\alpha_{\max 2}$ & Slip angle for the friction peak at load 2 & $8^{\circ}$ & $8^{\circ}$ \\
\hline$Q_{x}$ & Longitudinal shape factor & 1.9 & 1.9 \\
\hline$Q_{y}$ & Lateral shape factor & 1.9 & 1.9 \\
\hline
\end{tabular}

Next, the longitudinal and lateral friction coefficient in equation 13 and 14 are described by

$$
\begin{aligned}
& \mu_{x i}=\mu_{x i \text { max }} \sin \left(Q_{x} \arctan \left(\frac{\pi}{2 \arctan \left(Q_{x}\right)} \sqrt{\alpha_{n i}^{2}+\kappa_{n i}^{2}}\right)\right) \\
& \mu_{y i}=\mu_{y i \text { max }} \sin \left(Q_{y} \arctan \left(\frac{\pi}{2 \arctan \left(Q_{y}\right)} \sqrt{\alpha_{n i}^{2}+\kappa_{n i}^{2}}\right)\right)
\end{aligned}
$$

where $Q_{x}$ and $Q_{y}$ Are shaping factors.

By simplifying the tyre model in such way, the computation time for the OCP can be reduced while some important features of a race tyre remain to be described. This includes: 1) nonlinear tyre characteristics with respect to tyre slip, 2) variations of peak friction coefficients due to change of normal force, 3) combined slip and shaping factors for friction coefficients determination. It should be noted that in equation 13 and 14, a small value $\epsilon$ is added into the denominator of the combinedslip coefficient. This is a value to avoid zero-slip scenario leading to an infinite gradient derivative which will make the OCP solver to struggle. Meanwhile it should be small enough (10e-06) to maintain the accuracy of the tyre model.

For current Gen2 and future Gen3 cars, the driving wheels are propelled by torques from motors through LSD and drive shafts. The LSD torque is transferred from the faster rotating wheel to the slower wheel. This torque is given by:

$$
T_{\text {diff }}=0.5 k_{d}\left(\omega_{\text {inner }}-\omega_{\text {outer }}\right)
$$

where $\omega_{\text {inner }}$ and $\omega_{\text {outer }}$ are the wheel angular velocities of inner and outer wheel on the same axle and $k_{d}$ is the rotational damping coefficient of the LSD.

Finally, the wheel motions are defined by:

$$
J_{w} \dot{\omega}_{1}=k_{b} T_{\text {brake }}+T_{\text {diff } f}-F_{x 1} R
$$




$$
\begin{gathered}
J_{w} \dot{\omega}_{2}=k_{b} T_{\text {brake }}-T_{\text {diff } \_f}-F_{x 2} R \\
J_{w} \dot{\omega}_{3}=T_{\text {drive }}+\left(1-k_{b}\right) T_{\text {brake }}+T_{\text {regen } \_p}-T_{\text {diff_r }}-F_{x 3} R \\
J_{w} \dot{\omega}_{4}=T_{\text {drive }}+\left(1-k_{b}\right) T_{\text {brake }}+T_{\text {regen_p }}+T_{\text {diff_r }}-F_{x 4} R
\end{gathered}
$$

In which $J_{w}$ is the wheel rotational inertia, $k_{b}$ is the brake bias to the front, $T_{\text {drive }}$ and $T_{\text {brake }}$ are drive torque from the motor brake torque generated by the caliper or the motor regeneration. $T_{\text {regen_p }}$ is additional regenerative torque which in real life operated by driver on the steering wheel. To avoid overlapping of the pedals, these three torques are subject to the constraints of :

$$
\begin{aligned}
& T_{\text {drive }} T_{\text {brake }}=0 \\
& T_{\text {drive }} T_{\text {regen }}=0
\end{aligned}
$$

In this study, the $T_{d i f f_{-} f}$ is zero on Gen2 cars and non-zero for Gen 3 cars because the difference of powertrain configuration on front axle.

Therefore, the drive power $P_{\text {drive }}$ and regeneration power $P_{\text {regen }}$ are given by:

$$
\begin{gathered}
P_{\text {drive }}=\left(T_{\text {drive }}+T_{\text {diff }_{-} r}\right) \omega_{4}+\left(T_{\text {drive }}-T_{\text {diff } r}\right) \omega_{3} \\
\left.\left.P_{\text {regen }_{\text {gen } 2}=\left(k_{r}\left(1-k_{b}\right)\right.}\right) T_{\text {brake }}+T_{\text {regen }_{p}}+T_{\text {diff }}\right) \omega_{4}+\left(k_{r}\left(1-k_{b}\right) T_{\text {brake }}\right. \\
\left.+T_{\text {regen } p}-T_{\text {diff } f_{-} r}\right) \omega_{3}
\end{gathered}
$$

where $k_{r}$ denotes the portion of much of regenerative brake in total brake torque. For Gen 3 cars with front wheel MGU, the regeneration power becomes:

$$
\begin{gathered}
P_{\text {regen } \text { gen } 3}=\left(k_{r}\left(1-k_{b}\right) T_{\text {brake }}+T_{\text {regen }_{p}}+T_{\text {diff } f_{r}}\right) \omega_{4}+ \\
\left(k_{r}\left(1-k_{b}\right) T_{\text {brake }}+T_{\text {regen }}-T_{\text {diff } f_{r}}\right) \omega_{3}+ \\
\left(k_{r} k_{b} T_{\text {brake }}+T_{\text {regen_ } p}+T_{\text {diff } f}\right) \omega_{1}+\left(k_{r} k_{b} T_{\text {brake }}+T_{\text {regen_ } p}-T_{\text {diff } f}\right) \omega_{2}
\end{gathered}
$$

\subsubsection{Normal tyre loads}

The normal forces on the tyres have to be calculated at each time step to be used for calculation of the longitudinal and lateral tyre forces. These forces have to satisfy the basic balancing equations of the car. In the vertical direction:

$$
F_{z 1}+F_{z 2}+F_{z 3}+F_{z 4}-M g-F_{a z}=0
$$

where $F_{z i}$ are the tyre normal force on each tyre, $M$ is the total vehicle mass, $g$ is the acceleration due to gravity and $F_{a z}$ is the aerodynamic vertical load on the car. To balance the moment around the x-axis (illustrated in figure 3 ) of car:

$$
w\left(F_{z 4}-F_{z 3}\right)+w\left(F_{z 1}-F_{z 2}\right)+F_{y}=0
$$

and for the moment around the y-axis:

$$
b\left(F_{z 3}+F_{z 4}\right)-a\left(F_{z 1}+F_{z 2}\right)-h F_{x}-\left(a_{A}-a\right) F_{a z}=0
$$

in which $\mathrm{h}$ is the height of the vehicle mass center to the ground.

To ensure a unique solution for the four normal forces, the lateral load transfer bias $D$ is introduced to formulate the fourth balancing equation. This describe the lateral load transfer distribution between the front and rear axle:

$$
D\left(F_{z 2}+F_{z 3}-F_{z 1}-F_{z 4}\right)-F_{z 2}+F_{z 1}=0
$$

where the aero loads $F_{a z}$ and $F_{a x}$ are given by:

$$
\begin{gathered}
F_{a z}=0.5 C_{l} \rho_{a} A u^{2} \\
F_{a x}=0.5 C_{d} \rho_{a} A u^{2}
\end{gathered}
$$

in which $C_{d}$ and $C_{l}$ are the aerodynamic coefficient of drag and lift, $\rho_{a}$ is the air density and $A$ 
is the frontal area of the car.

\subsection{Thermal dynamics model}

The battery temperature is another important factor that should be taken into account in Formula-E energy management optimization. In particular races on the calendar, such as Santiago E-prix, the ambient and track temperature are so high that the battery temperature needs to be carefully managed to avoid derating in power.

In this study, the battery thermal behavior is modelled using a lump model. The heat generation of the battery is mainly composed of reversible heat and irreversible heat [30]. The irreversible heat mainly comes from the overpotential heat, material phase changes and mixing [31]. The overpotential heat is the main component which includes ohmic loss, charge transfer at the interface and mass transfer limitation, while in many studies, the heat generation due to phase change and mixing are often neglected [32].

The reversible heat is generated from entropy change in the cells. Bernardi [33] proposed the initial model of this component based on which different forms have been developed in the literature later. The reasons that both reversible heat and irreversible heat are both considered here are as follows: 1) the irreversible heat is dominant in high current applications [34] such as Formula-E application, 2) the reversible heat component significantly affects the battery temperature during discharging/charging cycles because of the change of current direction which happens very often on Formula-E cars due to regenerative brakes, and 3) the effect of change of state of charge (SOC) on reversible heat generation is obvious so the heat generation rate varies at different stage of a race i.e. different SOC.

The battery temperature $T_{b}$ is described by:

$$
m_{b} C_{b} \frac{d}{d t} T_{b}(t)=Q_{1}+Q_{2}-Q_{3}-Q_{4}
$$

in which $m_{b}$ is the battery total cell mass, $C_{b}$ is the specific heat capacity of the cell, $Q_{1}$ is the irreversible heat generation component, $Q_{2}$ is the reversible heat generation component, $Q_{3}$ is the conductive heat transfer rate from the cell mass to environment through the coolant and $Q_{4}$ is the radiative heat transfer rate. The heat flow is illustrated in figure 4.

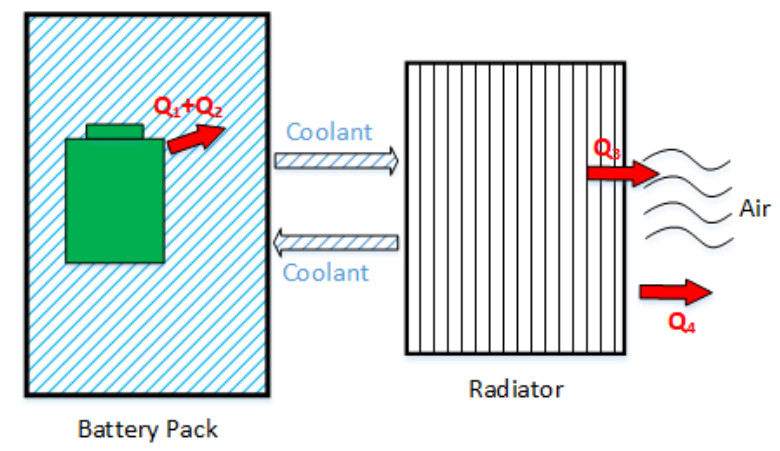

Figure 4: Schematic of heat flow used in battery thermal model in FE car

Because the only passive cooling device is the radiator and the rest of the system is covered under the vehicle body work, we consider the batteries and coolant as a whole. The $m_{b} C_{b}$ in eq.43 is added with $m_{c} C_{c}$ to include the coolant mass $m_{c}$ and specific heat capacity $C_{c}$.According to [35], 
the heat generation is given by:

$$
Q_{1}+Q_{2}=I\left(U_{o c_{-} b}-V_{b}\right)-I\left(T_{b} \frac{d U_{o c \_} b}{d T_{b}}\right)
$$

where $U_{o c_{-} b}$ is the open circuit voltage, $V_{b}$ is the terminal voltage, $I$ is the current. The term $\left(U_{o c_{-} b}-V_{b}\right)$ is caused by internal resistance:

$$
U_{\text {oc_ } b}-V_{b}=I\left(R_{p}+R_{o}\right)
$$

in which $R_{o}$ and $R_{p}$ are ohmic and polarization resistance of the battery which vary at different SOC. The term $\left(T_{b} \frac{d U_{o c, b}}{d T_{b}}\right)$ in equation 44 is often referred to as the entropy coefficient which is also SOC dependent. Therefore, the heat generation can be rewritten as follows:

$$
Q_{1}+Q_{2}=I^{2} R(S O C)-I T_{b}\left(\frac{d U_{o c_{b}}}{d T_{b}}\right)(S O C)
$$

In which $R(S O C)$ is the sum of polarization and ohmic resistance of the battery as a function of SOC and $\left(\frac{d U_{O c_{b}}}{d T_{b}}\right)(S O C)$ denotes the entropy coefficient which is also a function of SOC.

The heat transfer rate is given by:

$$
\begin{aligned}
& Q_{3}=h_{c} A_{b}\left(T_{b}-T_{a m b}\right) \\
& Q_{4}=\sigma \varepsilon_{e}\left(T_{b}^{4}-T_{a m b}^{4}\right)
\end{aligned}
$$

In which $A_{b}$ is the area of cell surface, $T_{a m b}$ is the ambient temperature, $h_{c}$ is the convective heat transfer coefficient, $\sigma$ is the Stefan Boltzmann constant and the $\varepsilon_{e}$ emissivity of the cell surface [36]. However, because the difference between the battery and environment is relatively low, the radiative heat transfer rate can be simplified [31] by linearization as:

$$
Q_{4}=4 \sigma \varepsilon_{e} T_{a m b}\left(T_{b}-T_{a m b}\right)
$$

Therefore the sum of convective and radiative heat transfer rate can be reformulated as:

$$
Q_{h t}=h_{c o m b}(V) A_{b}\left(T_{b}-T_{a m b}\right)
$$

where $h_{\text {comb }}$ is a combined heat transfer coefficient as a function to vehicle speed.

The battery and cooling data are obtained from authors' previous study [37] that is presented in appendix.

\section{Optimal control problem formulation}

The optimal control problem is formulated in order to minimize the following cost function in Bolza form:

$$
J=\varnothing\left(t_{0}, x\left(t_{0}\right), t_{f}, x\left(t_{f}\right), p\right)+\int_{t_{0}}^{t_{f}} l(t, x(t), u(t), p) d t
$$

which is subjected to these constraints:

$$
\left\{\begin{array}{c}
\frac{d x}{d t}-f(t, x(t), u(t), p)=0 \\
g(t, x(t), u(t), p)=0 \\
h(t, x(t), u(t), p) \leq 0 \\
g_{b}\left(x\left(t_{0}\right), x\left(t_{f}\right), u\left(t_{0}\right), u\left(t_{f}\right), p\right)=0
\end{array}\right.
$$

where the first term in equation 51 is the boundary (Mayor) cost and the second term is the stage (Lagrange) cost.

In the problem, $p \in R^{n_{p}}$ denotes the constant parameters to be optimized in the form of vector of $n_{p}$ dimensions, $x(t) \in R^{n}$ is the state vector and $\mathrm{u}(t) \in R^{m}$ is the control vector. The system 
dynamics is described by $f(t, x(t), u(t), p) \in R^{n}$, and vectors $g \in R^{n_{g}}$ and $g_{b} \in R^{n_{g b}}$ are the quality constraints whereas the latter defines the constraints of the boundaries. The inequality constraints are defined in $h \in R^{n_{h}}$.

In this study, the aim is to optimize the MTM along the track length which is an integration of $S f$ (i.e. reciprocal of velocity of vehicle along the track centerline), so the cost function will not contain the boundary cost but only the Lagrange stage cost which is:

$$
J=\int_{s_{0}}^{s_{t}}\left(S f+\epsilon \dot{u}^{T} W \dot{u}\right) d s
$$

where $W$ is a positive definite weight matrix, $s_{0}$ and $s_{f}$ are the initial and terminal length of the track respectively, $\dot{u}$ and $\dot{u}^{T}$ denote the change rate of control vectors and its transposed form. The term $\epsilon \dot{u}^{T} W \dot{u}$ is piece-wise derivative variation perturbation term which is added to the stage cost to regularize the problem. This is to avoid singular arcs and oscillatory in the solution caused by the nature of numerical algorithms [38]. Despite that this additional term would change the formulation to no longer a pure minimal time problem, a choice of a very small value for $\epsilon \in$ $(10 e-4,10 e-2)$ makes the influence to the solution negligible meanwhile it provides the benefit of a quicker convergence [39].

The system dynamics equations are included in $f(t, x(t), u(t), p)$, whereas the load transfer equations and equation for pedal overlapping constraint are treated as equality path constraints included in $g(t, x(t), u(t), p)$. And finally, the bounds for states and controls, and path constraint for power are included in the inequality constraints $h(t, x(t), u(t), p)$. Table 35 details the elements in the formulation.

Table 3: OCP formulation elements and their physical meanings

\begin{tabular}{|c|c|c|}
\hline Components & Elements & Physical meaning \\
\hline Lagrange stage cost & $S f+\epsilon \dot{u}^{T} W \dot{u}$ & $\begin{array}{l}S f \text { (i.e. reciprocal of velocity of vehicle } \\
\text { along the track centerline) and } \\
\text { regularization } \epsilon \dot{u}^{T} W \dot{u}\end{array}$ \\
\hline Change rate of control vectors & $\dot{u}$ & $\begin{array}{l}\text { Including pedal change rate of steering } \\
\text { angle, acceleration pedal, braking pedal } \\
\text { and regeneration pedal. }\end{array}$ \\
\hline System dynamics & $f(t, x(t), u(t), p)$ & $\begin{array}{l}\text { Equations describing the vehicle } \\
\text { dynamics, track positions and battery } \\
\text { thermal dynamics }\end{array}$ \\
\hline Equality constraints & $g(t, x(t), u(t), p)$ & Load transfer equations (eq. $37,38,39,40$ ) \\
\hline The inequality constraints & $h(t, x(t), u(t), p)$ & $\begin{array}{l}\text { Constraints of states (e.g. vehicle lateral } \\
\text { position), controls (e.g. steering angle). } \\
\text { Path constraints (e.g. MGU power) and } \\
\text { boundary constraints (e.g. energy } \\
\text { consumption) }\end{array}$ \\
\hline
\end{tabular}

The optimal control problem in this study is transcribed into a large NLP using Legendre-GaussRadau (LGR) quadrature orthogonal collocation method and Radau's integration formula [40]. To implement this, the General-Purpose Optimal Control MATLAB Toolbox GPOPS-II [41] and underlying interior point algorithm IPOPT [42] are used. A ph-method for mesh refinement [43] is 
used to reduce the polynomials approximating errors which will further reduce the influence of the problem characteristics (e.g. singular arcs, rapidly changing controls and constraints). Similar problems and detailed discussion of such collocation and integration methods can be found in [44]. Scaling is another factor that can significantly affect the convergence performance of the optimization algorithms. Instead of simply scaling into the range of $[-0.5,0.5]$, to map the variables into a more spherical space[38], the base quantities are scaled into dimensionless quantities as shown in table 4.

With this method, the vehicle mass of $900 \mathrm{~kg}$ now is dimensionless value of 1 . Other derived quantities such as power of $250 \mathrm{~kW}$ now is 5.14 , tyre normal force of $6000 \mathrm{~N}$ now is 0.68 and 50000 $\mathrm{J}$ of energy is scaled to 1.8 , etc.

Table 4: Non-dimensional scalers, which are used in the optimization algorithms

\begin{tabular}{|l|l|l|}
\hline Base quantity & Unscaled (with unit) & Scaled (dimensionless) \\
\hline Mass & $1 \mathrm{~kg}$ & 0.001111111 \\
\hline Length & $1 \mathrm{~m}$ & 0.322580645 \\
\hline Time & $1 \mathrm{~s}$ & 1.778001778 \\
\hline Electric current & $1 \mathrm{~A}$ & 0.005 \\
\hline Thermaldynamic temperature & $1 \mathrm{~K}$ & 0.05 \\
\hline
\end{tabular}

\section{Result and discussion}

\subsection{Reference performance}

This section introduces two performance references whose energy consumption and thermal behavior are not restricted. The power out of RESS is restricted to $200 \mathrm{~kW}$ (race mode) for the first reference and $250 \mathrm{~kW}$ (qualification mode) for the second reference. The key differences are shown in Table 5.

Table 5: Performance references whose energy consumption and thermal behavior are not restricted

\begin{tabular}{|l|l|l|l|l|}
\hline Case & $\begin{array}{l}\text { Power } \\
(\mathrm{kW})\end{array}$ & $\begin{array}{l}\text { Energy } \\
(\mathrm{kWh})\end{array}$ & $\begin{array}{l}\text { consumption } \\
(100 \%)\end{array}$ & $\begin{array}{l}\text { Temperature rise }\left({ }^{\circ} \mathrm{C}\right) \\
\text { Tenv }\left(25^{\circ} \mathrm{C}\right) \quad \mathrm{SOCi}^{*}\end{array}$ \\
\hline Ref 1 & 200 & 2.03 & 1.558 & 80.48 \\
\hline Ref 2 & 250 & 2.26 & 2.071 & 78.57 \\
\hline
\end{tabular}

Tenv* and SOCi* denote the environment temperature and initial SOC respectively

Figure 5 shows the track layout and race lines. The difference in power doesn't affect the race line much. The biggest difference occurred near turn 2 with maximum difference of $0.5 \mathrm{~m}$ shown in figure 5(b) and (d). In the majority parts of the track, the race lines are very close. In contrast, the higher power is generated in Ref $2 \mathrm{car}$, the more advantage is seen over speed of $50 \mathrm{~m} / \mathrm{s}$ as shown in figure 5(c). As a result, this has improved the lap time of the Ref 2 car by $1.91 \mathrm{~s}(2.37 \%)$ faster than the Ref 1 car. However this has also led to an extra energy consumption of $0.23 \mathrm{kWh}(11.3 \%)$. It should be noted that such a higher energy consumption did not significantly improve the lap time. 
In terms of thermal behavior, with $50 \mathrm{~kW}$ more power in the qualification mode, the battery temperature rises higher in the Ref 2 case. Since the thermal behavior is more complex to be analyzed under different initial and environment conditions, this is discussed in details in the following sections.
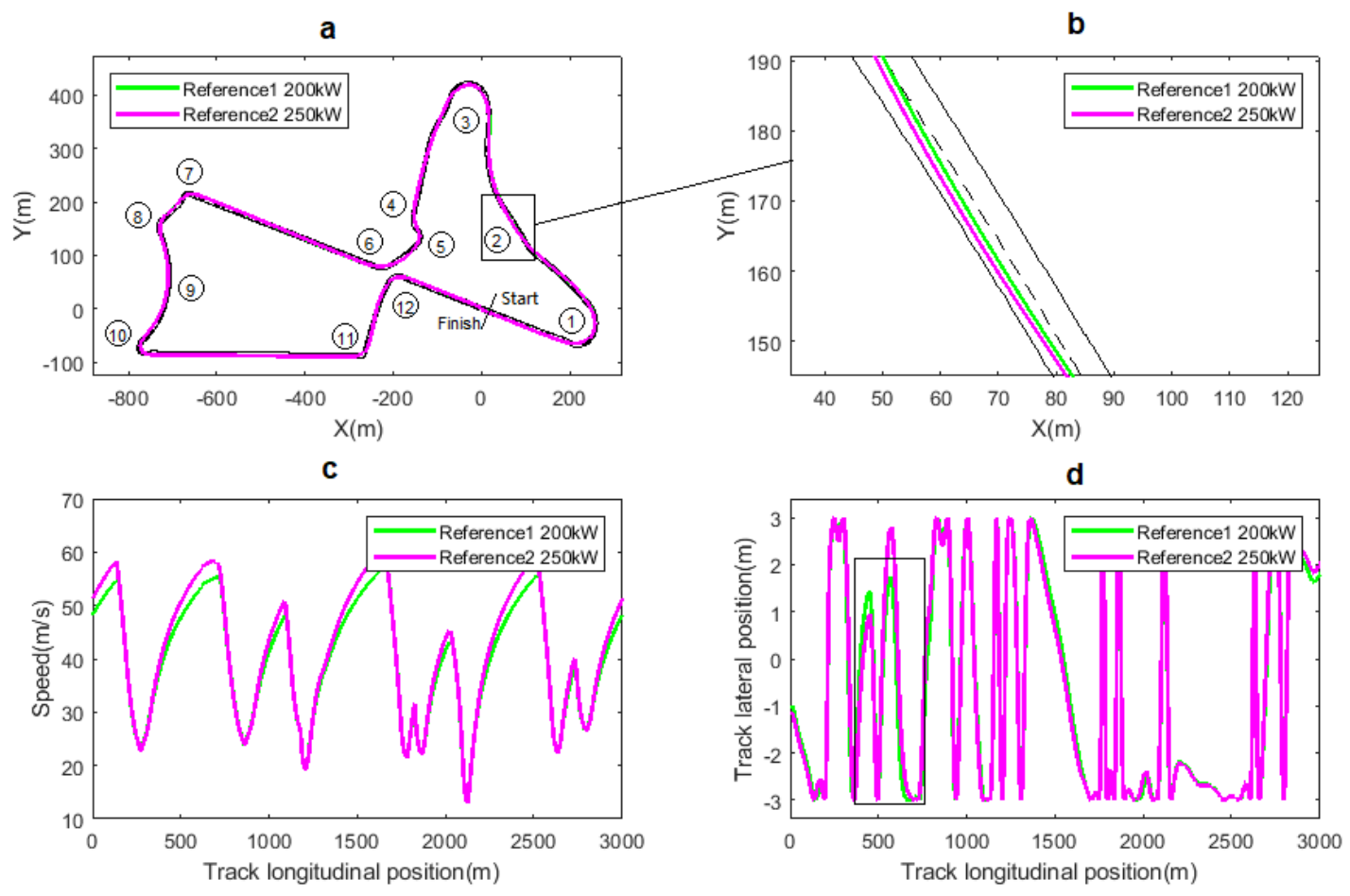

Figure 5: Lap time simulation: a) track layout and race lines, b) enlarged view of turn 2, c) speed profiles, d) lateral positions of the vehicle to the centreline

\subsection{Performance with limited energy}

This section demonstrates the effect of energy restriction on the vehicle's performance. Figure 6 shows how the energy restriction affects the speed on the track. It can be observed that given less available energy, the optimal solution for minimum lap time cuts more on the high-speed sections (blocks shown in figure 6). The car starts to slow down before reaching the possible high speed. The deceleration during the high-speed cut-off (arrow A in figure 6) is lower than the brake phase (arrow $\mathrm{B}$ in figure 6). This is because the former one is due to the combined effect of aerodynamic drag and rear axle regenerative torque by operating the regenerative pedal on the steering wheel as mentioned in Section 2.2.2. While in the latter, steeper deceleration is achieved by directly applying torque on both front and rear axles. The torque differences are shown in figure 7. 


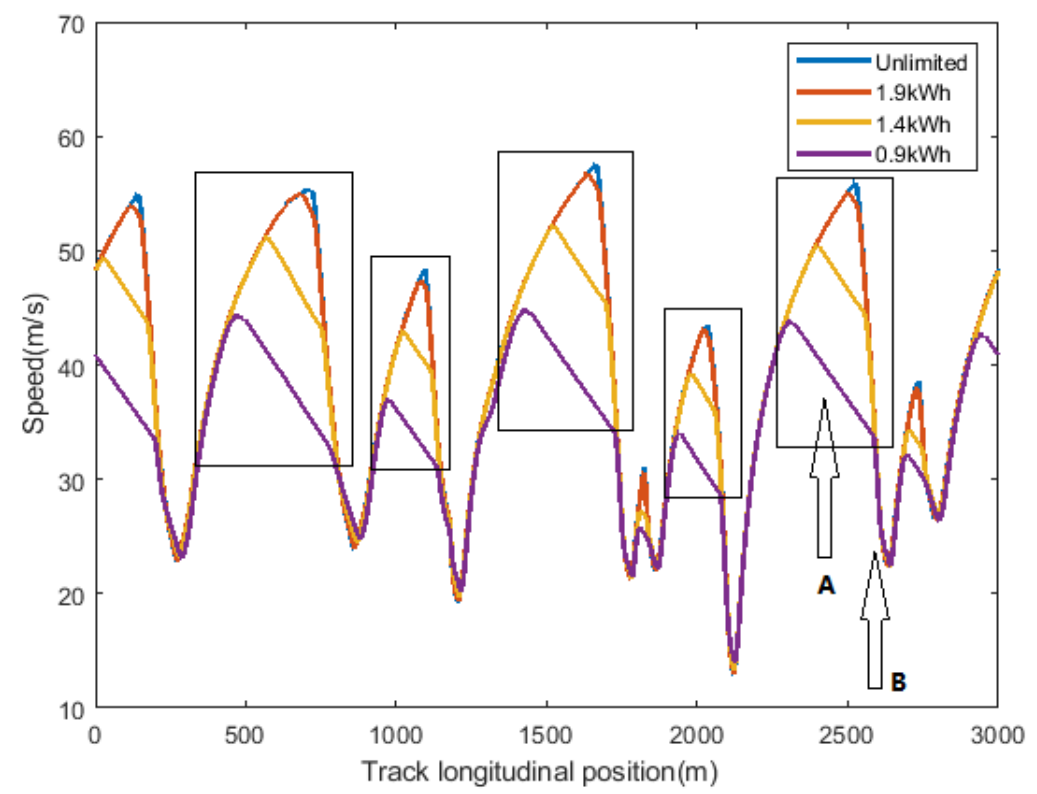

Figure 6: Speed profiles with different energy consumption limitations
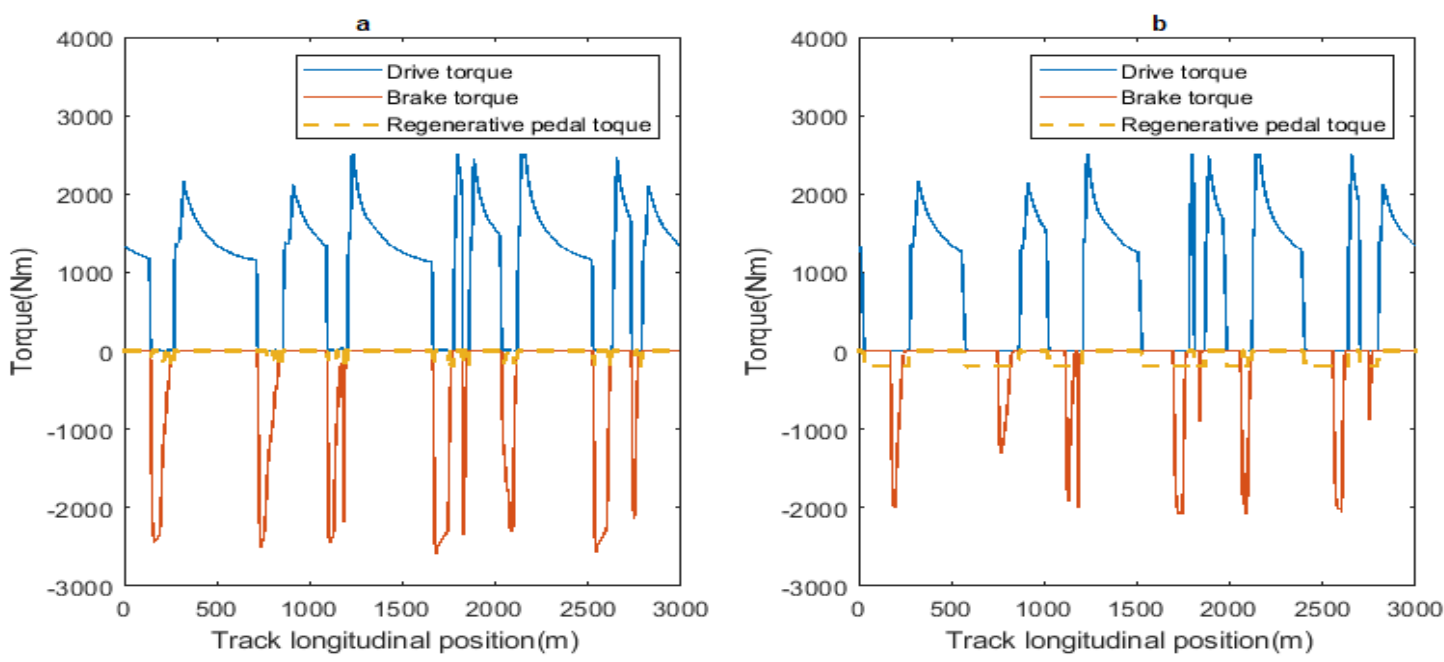

Figure 7: Drive, brake and regenerative pedal torques : a) unlimited energy case, b) restricted energy consumption to $1.4 \mathrm{kWh}$ per lap

It can be observed that in the unlimited case, brake torque is immediately applied after drive torque becomes zero (driver lifts his foot off the acceleration pedal). In contrast, when given an upper limit of energy consumption, there's an observable gap between the drive and brake torques which suggests that neither the acceleration pedal nor the brake pedal is pressed. This technique is referred to 'lift and coasting' (referred as $\mathrm{LaC}$ later in this paper), by which the car is slowing down on its momentum due to aerodynamic drag and energy is harvested by the additional regenerative torque. The amount of $\mathrm{LaC}$ increases as the energy consumption is restricted to a lower value as shown in figure 8(b). The slowing down before corner entry relies more on $\mathrm{LaC}$ than conventional braking when comparing figure 8(b) and (d). When the energy-per-lap is restricted to less than $1.4 \mathrm{kWh}$, the car is under $\mathrm{LaC}$ state for more than half of the distance of the track. Also, it should be noted that in the unlimited case, the regenerative pedal is still used, the purpose of this operation aims no longer for energy saving but as a way to alter the brake bias in order to enhance the corner entry brake 
performance. From the previous vehicle model(equation 8,11 and 42) and the result presented in figure 7, the reasons why $\mathrm{LaC}$ technique is an effective way to save energy can be explained in two aspects: 1) spending energy to gain higher speed advantage in high-speed sections is very inefficient because the race car hardly accelerates in those sections due to high aerodynamic drag. Therefore, energy consumption at such a high rate can only give very little time advantage; 2) $\mathrm{LaC}$ reduces energy cost in those high-speed sections and harvests energy from rear axle. This is different from stepping the brake pedal which in the meantime causes waste of energy on front axle through heat dissipation.
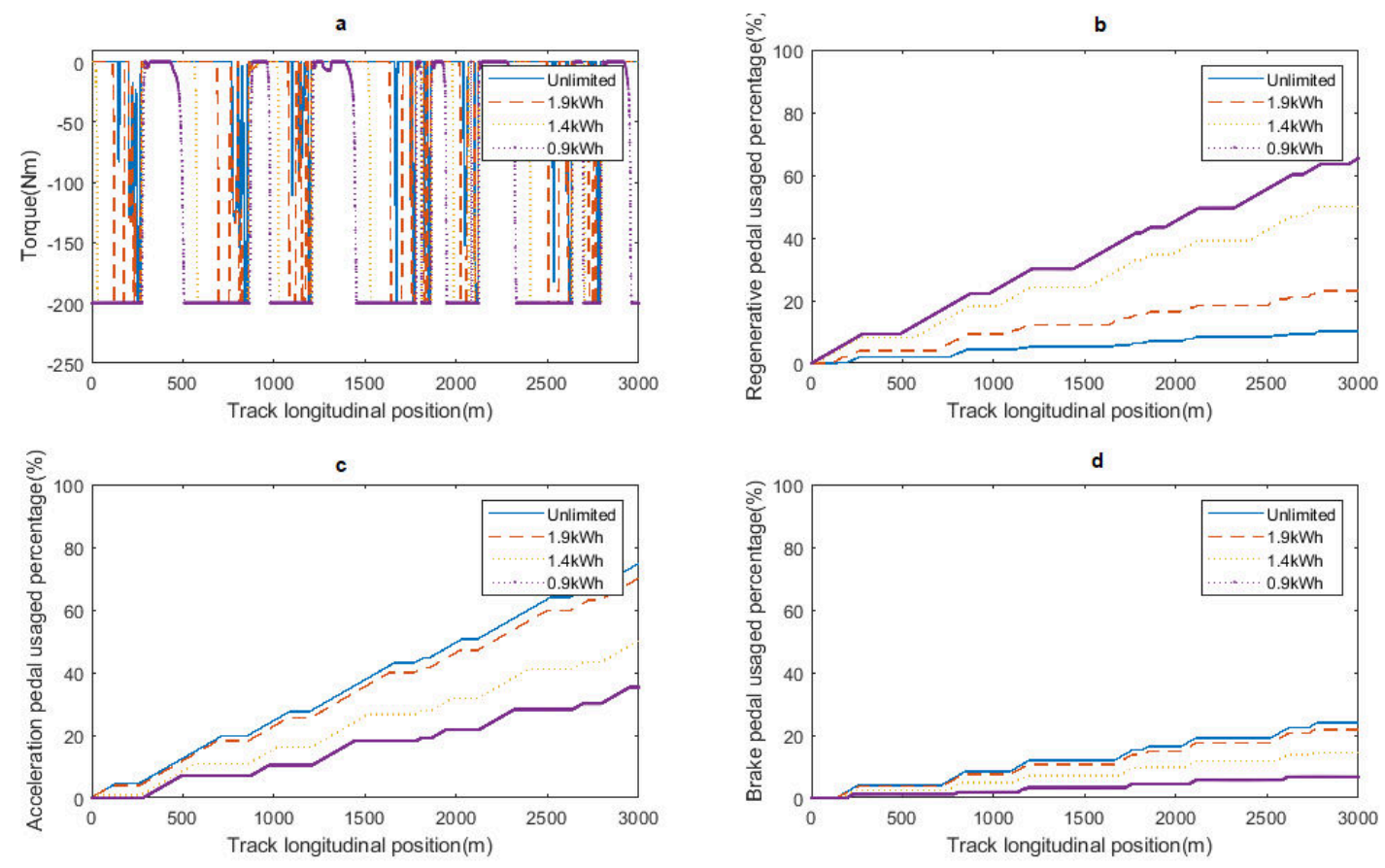

Figure 8: lap time simulation: a) torque applied by regeneration pedal, b) regeneration pedal usage throughout a lap, c) acceleration pedal usage, d) brake pedal usage

The energy restriction also has a minor effect on the choice of racing lines solved by the OCP. As shown in figure 9 , the influence lies in some specific parts of the track, enlarged in figure 9 (b),(c) and (d). The racing line with less energy is usually wider than the one with more energy. This can help to reduce the speed loss due to tight corners.

It is obvious that with a limited energy, the pace will be compromised; the effect of energy consumption limit on lap time is shown in figure 10. It is shown in the figure that the relation between energy-per-lap and lap time follows a very regular smooth polynomial curve:

$$
t_{\text {lap }}=2.717 E_{p}^{4}-20.53 E_{p}^{3}+64.13 E_{p}^{2}-97.43 E_{p}+139.6
$$

where $E_{p}$ is the restricted energy per lap and $t_{\text {lap }}$ is the resultant lap time.

It should be noted that such a relation is crucial for management for multiple laps which is too large for an OCP. The regulation states that a race lasts for 45 minutes plus one lap. With this relation, the time of 45 minutes can be converted to number of laps along with an energy-per-lap target. Then the race strategy can me formulated into a multi-stage problem. 

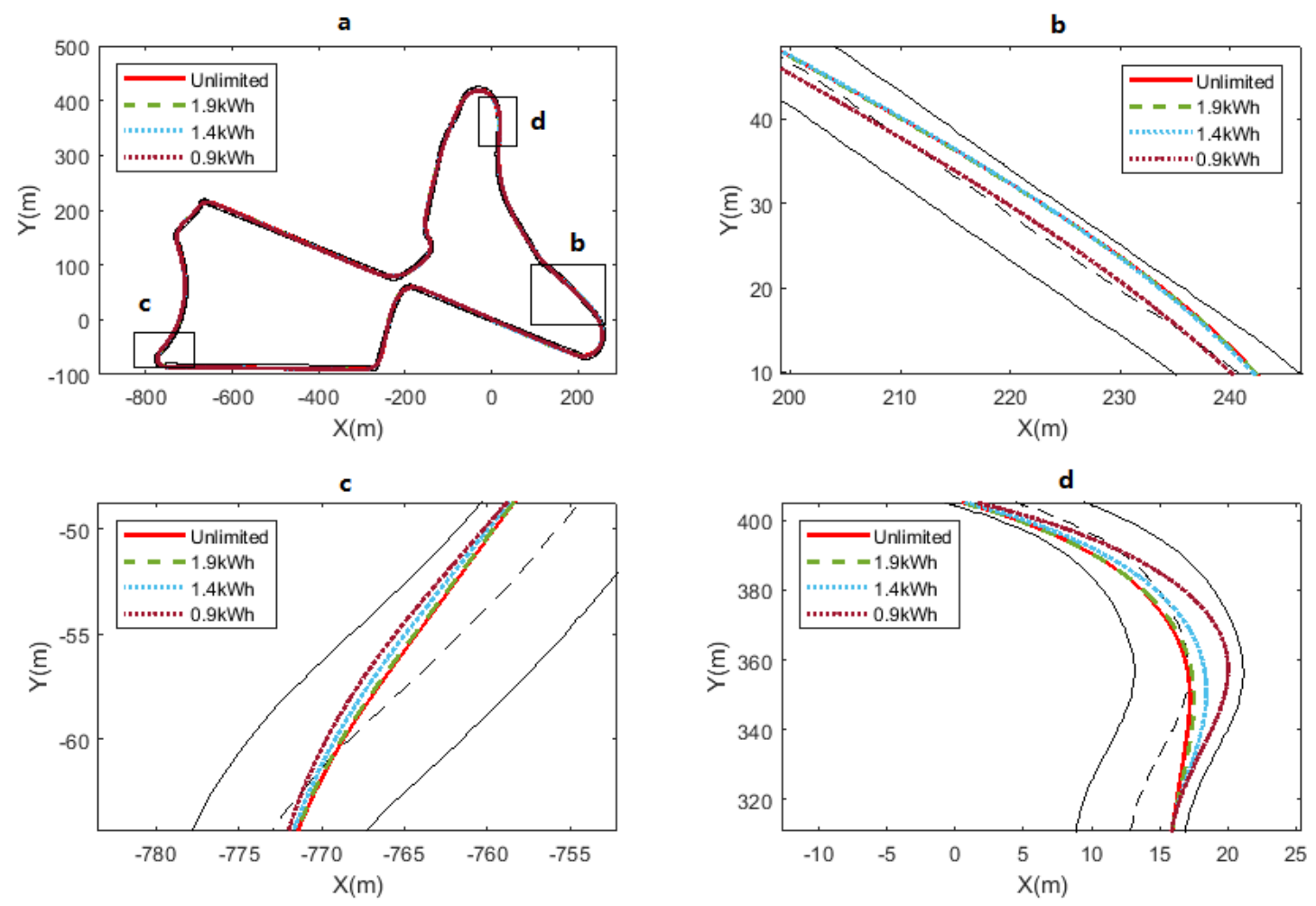

Figure 9: Racing lines in a lap time simulation: a) track-size view of race lines, b) enlarged view of block b, c) enlarged view of block c, and d) enlarged view of block $d$

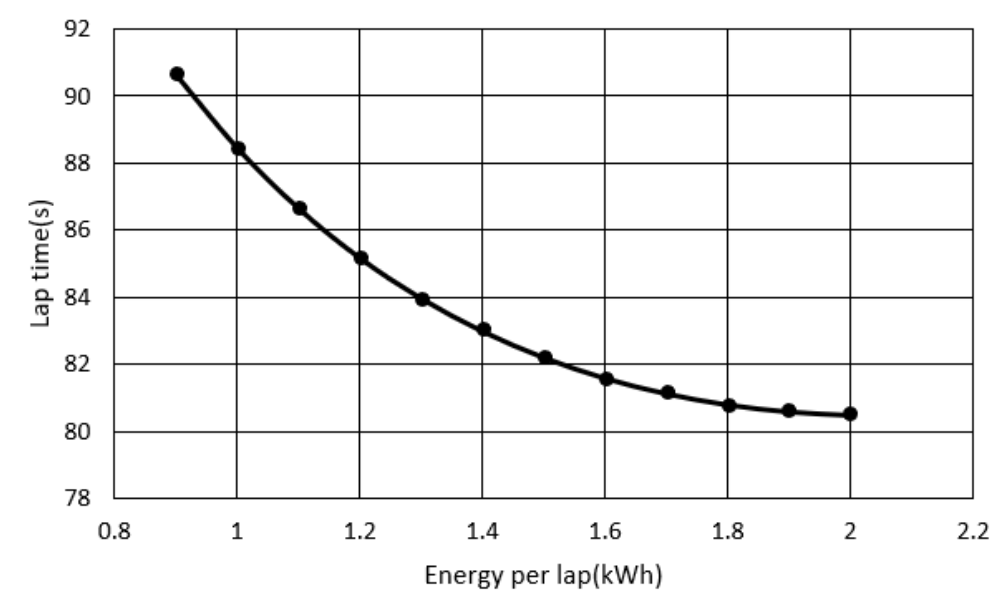

Figure 10: lap time as a function of energy consumption

\subsection{Battery thermal behavior analysis and its effect as a constraint}

\subsubsection{Unconstrained cases}

It is obvious that with more energy consumed, the battery temperature rises higher (shown in figure 11). However, the battery thermal behavior is more complex because it is affected by many factors such as initial conditions (e.g. initial battery temperature, SOC) and environment temperature. The most dominant factor is the battery SOC because the battery properties change with it such as 1) open circuit voltage (OCV), 2) polarization and ohmic resistance values, and 3) entropy coefficient. 
Three representative cases whose initial SOCs are $100 \%, 50 \%$ and $10 \%$, are investigated here. All of which are under the same $1.6 \mathrm{kWh}$ energy per lap restriction with initial temperature of $25^{\circ} \mathrm{C}$, which is a common ambient temperature when a race is held around March in Merrakesh (i.e. the track used in this study). Figure 12 shows the irreversible (Qirr) and reversible (Qrev) heat generation rate of the three cases. The first observed outcome is that the general magnitude of the heat generation rate is higher at lower SOCs. This is due to the characteristic of OCV versus SOC where more current is required when OCV drops at lower SOC given the same output power of RESS. The RESS current signal is shown for all the three cases in figure 13.

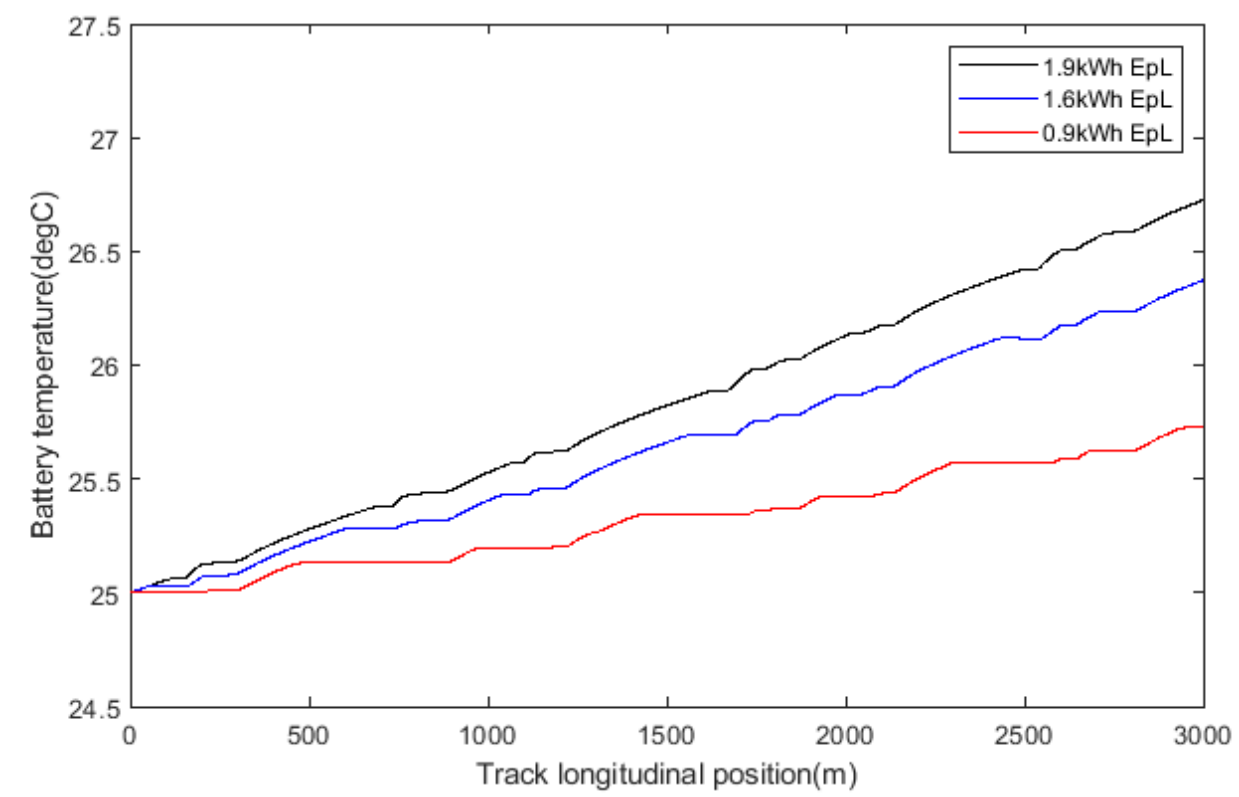

Figure 11: Battery temperature change at $25^{\circ} \mathrm{C}$ environment temperature with initial battery temperature at $25^{\circ} \mathrm{C}$ and $\mathrm{SOC}$ at $95 \%$ under different energy restrictions

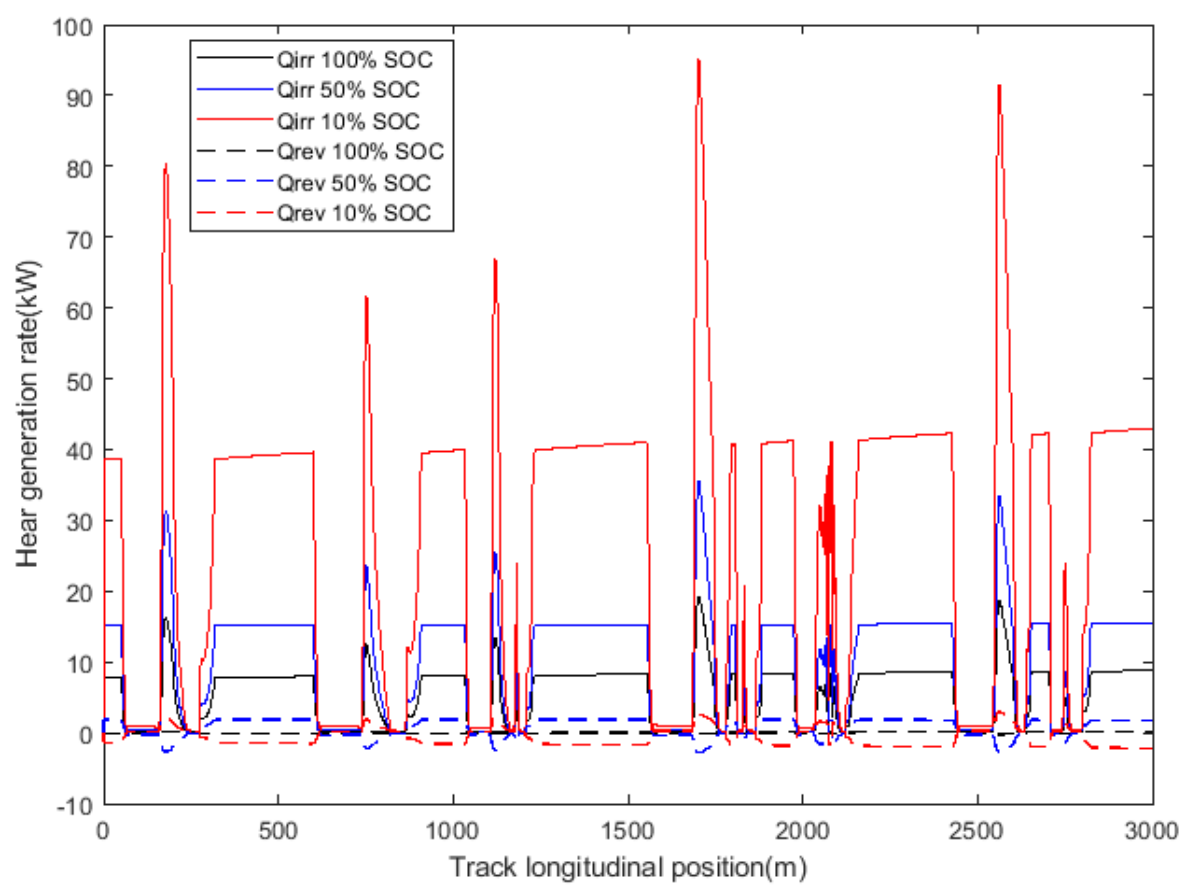

Figure 12: Battery heat generation rate under different SOC conditions 


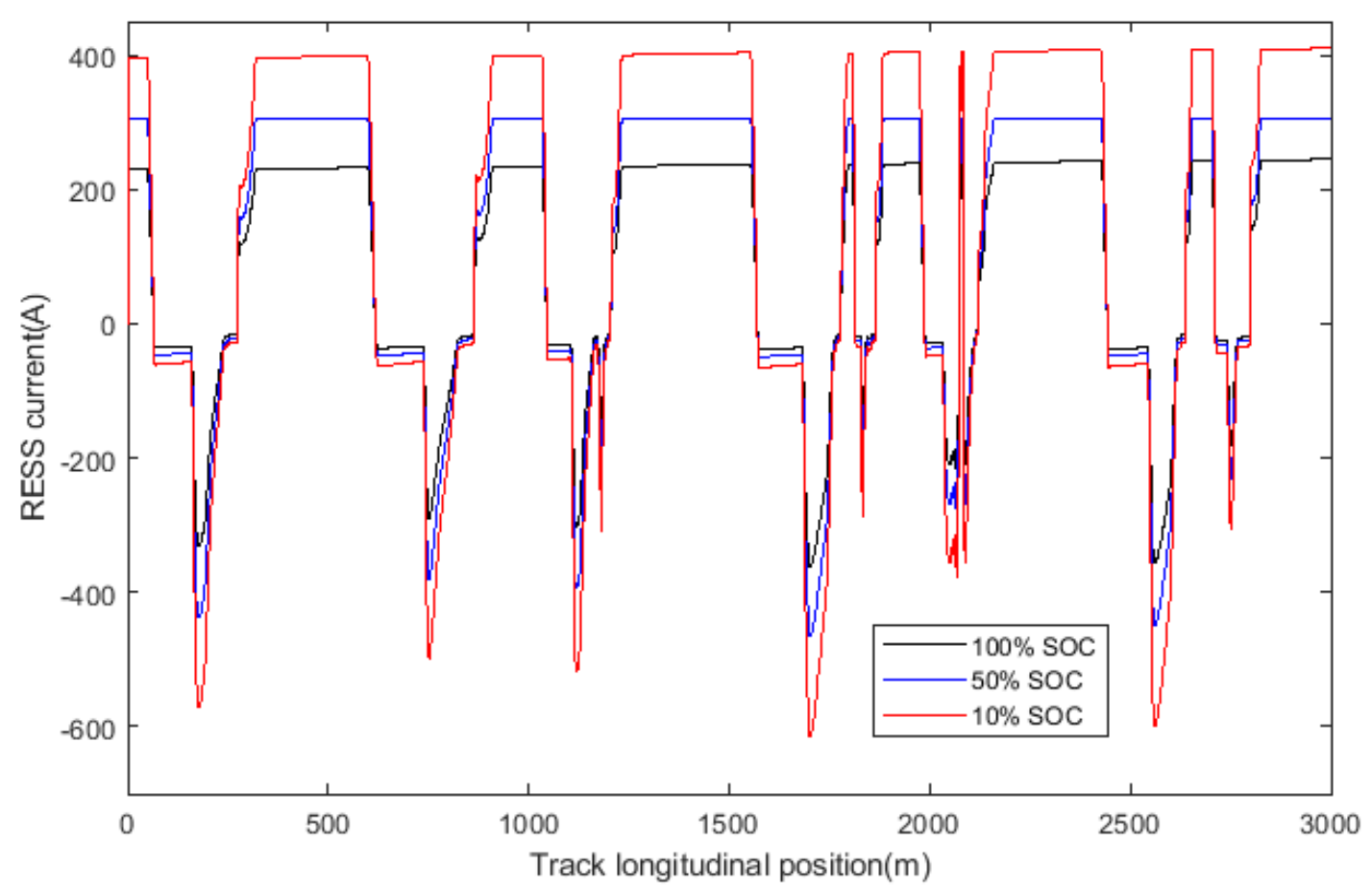

Figure 13: Current of the RESS under different SOC conditions

As is shown in figure 13, the current is higher in magnitude at lower SOC during both discharge (positive) and charge (negative). The combined effect of current and entropy coefficient also lead to changes in magnitude of different heat components. One feature of interest in figure 12 is that the signs of irreversible heat and reversible heat isn't the same at all time. This is because the change of entropy coefficient at different SOCs. At SOC of $50 \%$, the entropy coefficient is positive (shown in appendix) which means during regeneration the reversible component contributes to lower the total heat generation rate whereas during propelling, it increases the total rate. When the entropy coefficient is negative such as at SOC of $100 \%$ and $10 \%$, the effect is opposite. The battery temperature results also show big difference. Starting with an initial battery temperature of $35{ }^{\circ} \mathrm{C}$, the $10 \%$ SOC case resulted in a $4.8^{\circ} \mathrm{C}$ temperature rise which is 9 times higher compared to the $0.5^{\circ} \mathrm{C}$ rise with $100 \%$ SOC. However, in real life, this wouldn't happen because lower SOC always happen in the later phase of a race where battery temperature is already relatively high. This increases the temperature difference between the battery and ambient temperature which raises the cooling power (equation 50). As shown in figure 14, when the initial temperature is changed to a more realistic value, e.g. $55^{\circ} \mathrm{C}$, the temperature rise is decreased to $3.5^{\circ} \mathrm{C}$. 

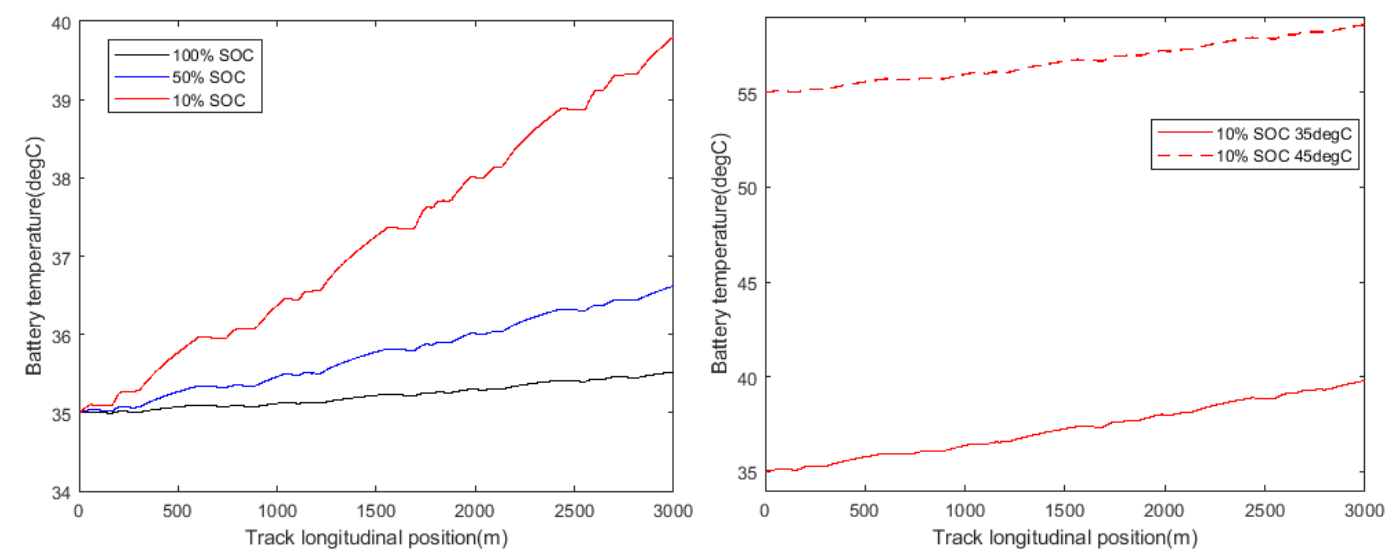

Figure 14: Battery temperature change: a) different SOCs starting at same temperature $35^{\circ} \mathrm{C}, \mathrm{b}$ ) same SOC $(10 \%)$ starting at $35^{\circ} \mathrm{C}$ and $55^{\circ} \mathrm{C}$

The following conclusions can be drawn from the unconstraint cases: 1) during a race, the heat generation is expected to be higher in the later phase due to the battery voltage drop, 2) in a certain range of SOC, the entropy coefficient is positive and harvesting energy will bring less heat generation. Therefore, more regeneration would cost less thermal penalty than a case where entropy coefficient is negative, and 3) the temperature difference between the battery and environment significantly influence the battery temperature rise. In places like Santiago where ambient temperature is high, thermal management would become more crucial. An example is discussed in the next section.

\subsubsection{OCP solution with thermal constraints}

This section demonstrates the OCP solution with thermal constraints added to a base energy constraint of $1.6 \mathrm{kWh}$ per lap. The initial battery temperature and SOC is set to $30^{\circ} \mathrm{C}$ and $100 \%$ respectively with ambient temperature of $25^{\circ} \mathrm{C}$. Figure 15 shows battery temperature change under different constraints. The black line in the figure shows the reference case with no limit on battery temperature rise. The temperature rise is $0.82^{\circ} \mathrm{C}$ finishing at a lap time of $81.59 \mathrm{~s}$. The blue and red lines show the cases with maximum temperature rise of $0.67^{\circ} \mathrm{C}$ and $0.48^{\circ} \mathrm{C}$ respectively. The corresponding lap times are $81.89 \mathrm{~s}$ and $83.01 \mathrm{~s}$. Given such initial conditions, the lap time sensitivity to temperature follows the pattern shown in figure 16. 


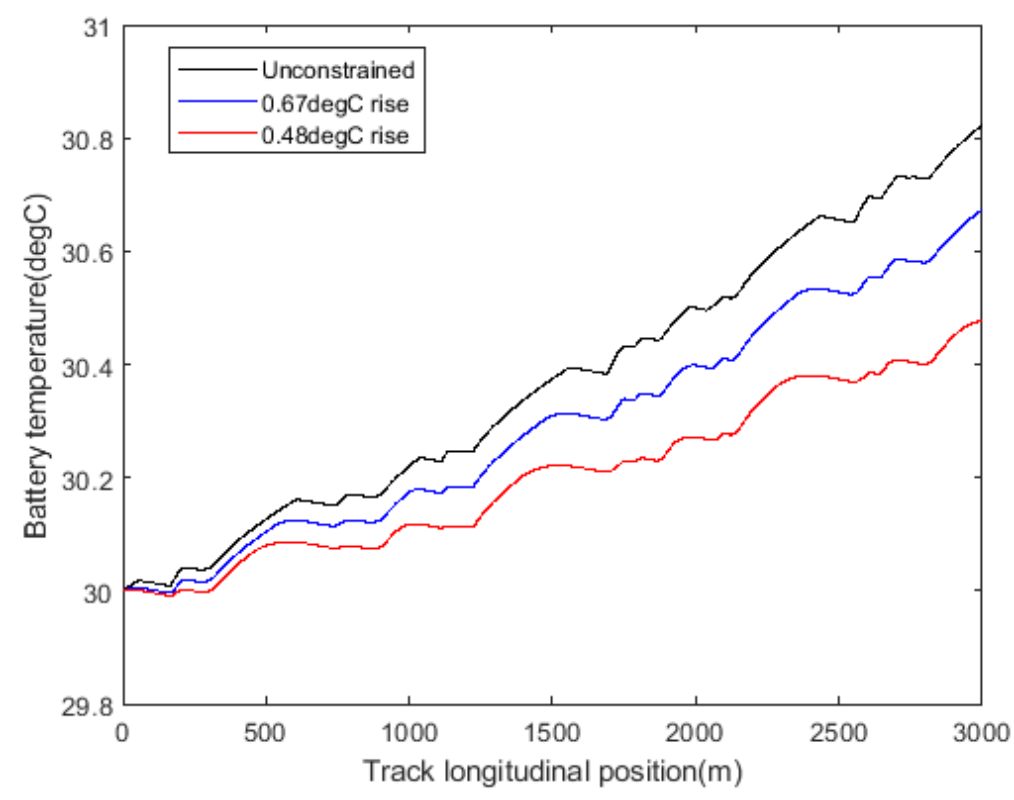

Figure 15: Battery temperature change under different constraints

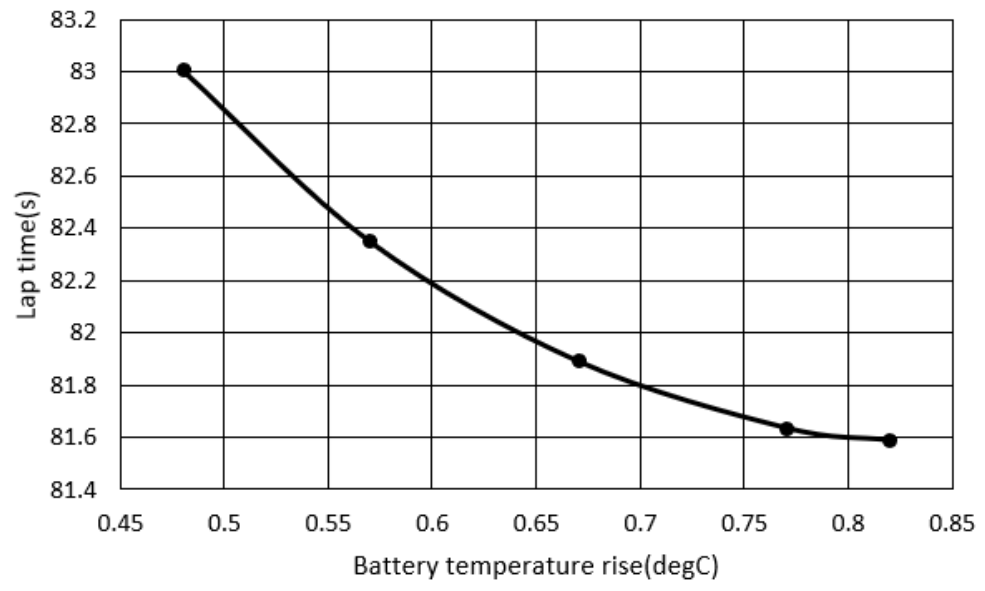

Figure 16: Lap time as a function of battery temperature rise

In the formulation of the OCP, the energy consumption is treated as an upper bound which means if other constraints are more dominant, the total given amount of energy doesn't need to be fully consumed. Empirically, to reduce temperature rise, the most direct way is to reduce the energy consumption. However, from the OCP result, every solution fully consumed the available $1.6 \mathrm{kWh}$ of energy. Therefore, the OCP produced a more efficient technique to reduce temperature rise. This is shown in figure 17. For the unlimited temperature case, the typical $\mathrm{LaC}$ technique can be clearly observed as previously discussed. The solution suggested an immediate complete lift off at certain points. When temperature constraints are applied, a clearly different style drive torque curve from the typical $\mathrm{LaC}$ can be observed. Instead of a sharp lift off, the solutions suggested a more gentle decrease of the drive torque (blocks shown in figure 17). It is observed that the more strict the temperature constraint is, the more gentle the decrease in drive torque is. This brings the starting point more advance and delays where it decreases to 0 . The effect of such technique on speed and heat generation is shown in figure 18 . 
For the unlimited case, when lift and coasting is performed, the speed profile changes sharply. In contrast, the constrained cases showed very smooth speed profiles which is unusual in race cars. The solution suggested to cut out some parts of high-speed performance while braking actions are not compromised. This decreases the amount of heat generated at those high-speed sections as can be observed in figure 17(b) to reduce the total temperature rise. The results in this section demonstrate a novel type of solution to thermal constraints compared to energy constraints.

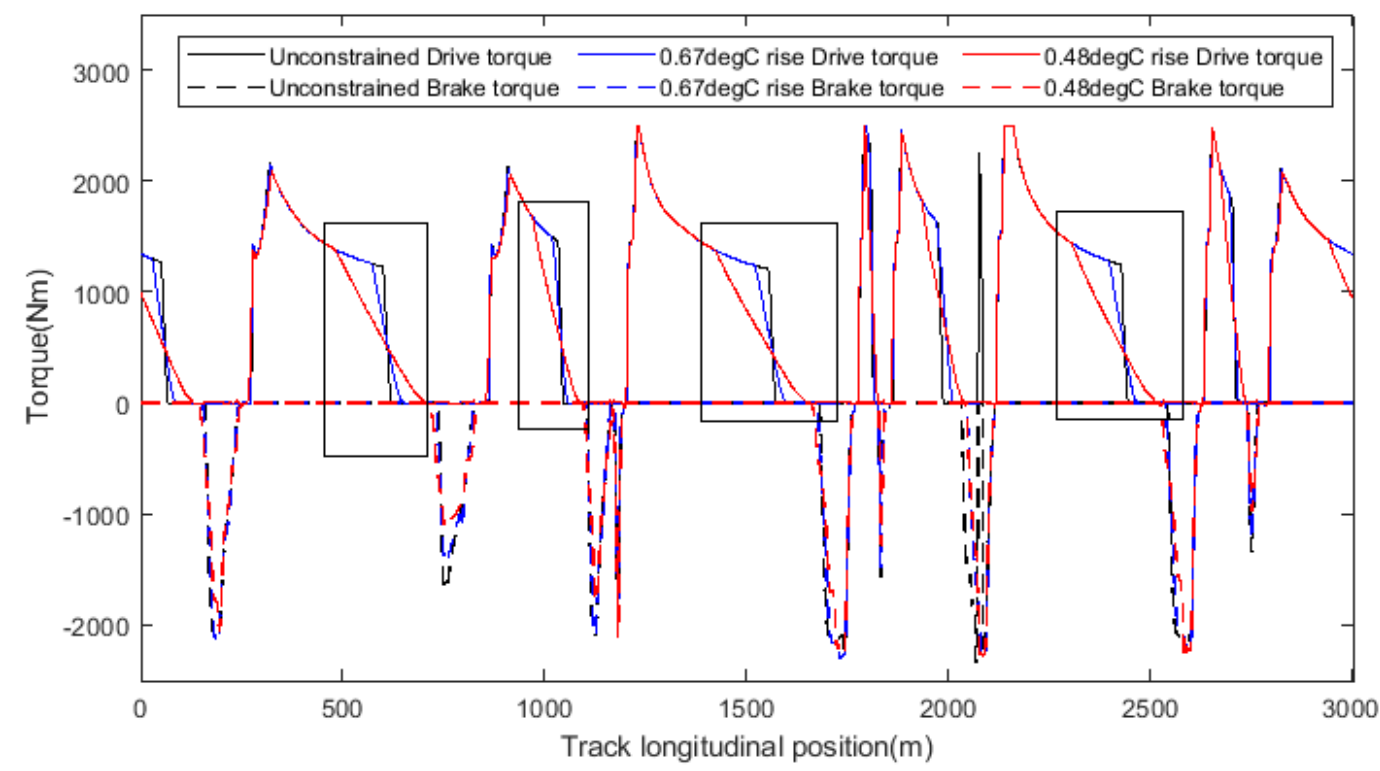

Figure 17: Drive and brake torques during lap time simulation under different battery thermal constraints
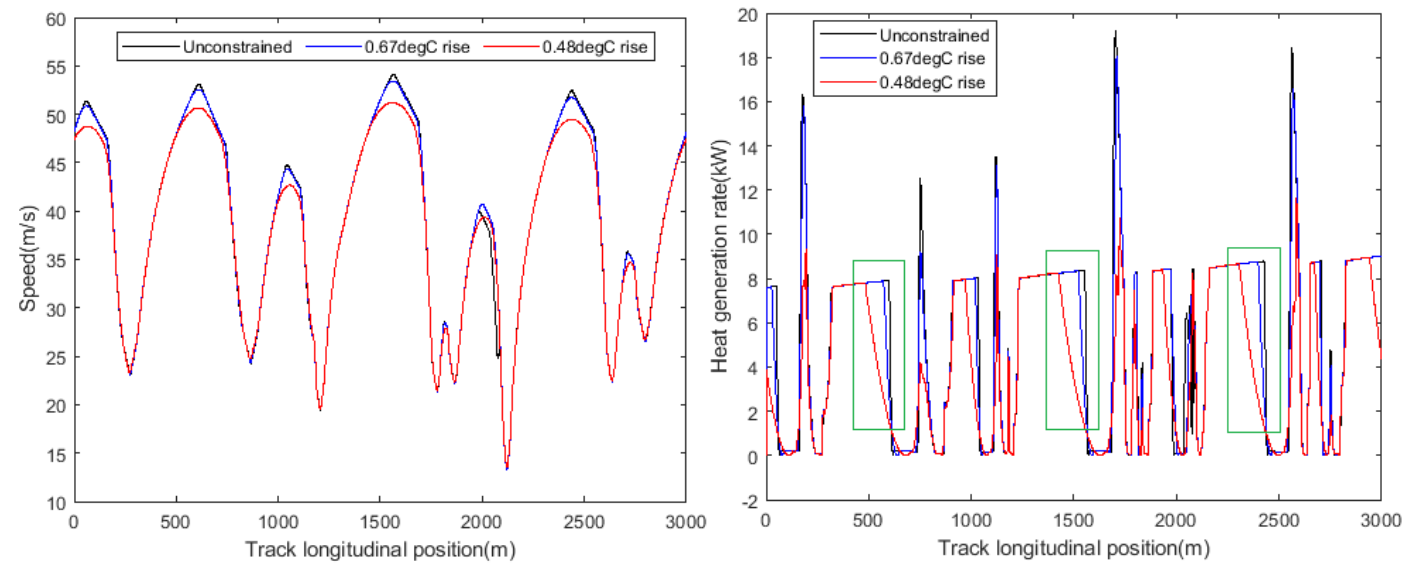

Figure 18: lap time simulation under different battery thermal constraints: a) speed profile, b) heat generation rate

\subsubsection{Battery resistance investigation}

As previously introduced in Section 2.3, the combined ohmic and polarization resistance of the battery in this study is assumed to be a function only of battery SOC (eq.46). However in real life, the characteristic of the resistance is usually more complex and resistance can change with battery temperature [45] and battery state of health [46]. This feature is strongly non-linear and sometimes 
non-monotonical with increase/decrease magnitude over 50\% [50]. While the detailed data are not easily accessible, as an alternative way, we have investigated the effect of resistance changes on the FE race strategy performance in this section by assuming changes of $-50 \%$ and $+20 \%$ in battery combined resistance. The decreased resistance is assumed to be the result of battery temperature rise and the increased resistance could be result of either temperature rise or aging. The initial condition for this investigation is set to $50 \% \mathrm{SOC}$ and battery temperature of $43^{\circ} \mathrm{C}$ which is a very likely state in a race suggested by [41]. The battery temperature rise is constrained to $1.5^{\circ} \mathrm{C}$ and energy consumption is restricted to $1.6 \mathrm{kWh}$. The key summary is shown in table 6 .

Table 6: Comparison between Gen 2 and Gen 3 simulation models

\begin{tabular}{|l|l|l|l|}
\hline Case & 1 & 2 & 3 \\
\hline Resistance & $\mathrm{R}(\mathrm{SOC})$ & $\mathrm{R}(\mathrm{SOC})+20 \%$ & $\mathrm{R}(\mathrm{SOC})-50 \%$ \\
\hline Terminal battery temperature $\left({ }^{\circ} \mathrm{C}\right)$ & 44.5 & 44.5 & 44.4185 \\
\hline $\begin{array}{l}\text { Peak heat generation rate during } \\
\text { propulsion }(\mathrm{kW})\end{array}$ & 16.97 & 19.99 & 9.46 \\
\hline Lap time(s) & 83.8975 & 84.8694 & 81.6203 \\
\hline
\end{tabular}

The first noticeable difference is the temperatures at the end of the lap. Case 3 with $50 \%$ reduced resistance did not reach the $1.5^{\circ} \mathrm{C}$ constraint of battery temperature rise while the other two reached the limit. This indicates that Case 3 is not thermal-constrained. As a result, the lap time is clearly faster than the first two cases. Also it can be seen that the peak heat generation rate during propulsion is nearly proportional to the resistance. This is because the dominant component of the total heat generation is the irreversible heat, which is proportional to the battery combined resistance. The detailed results are shown in figure 19.
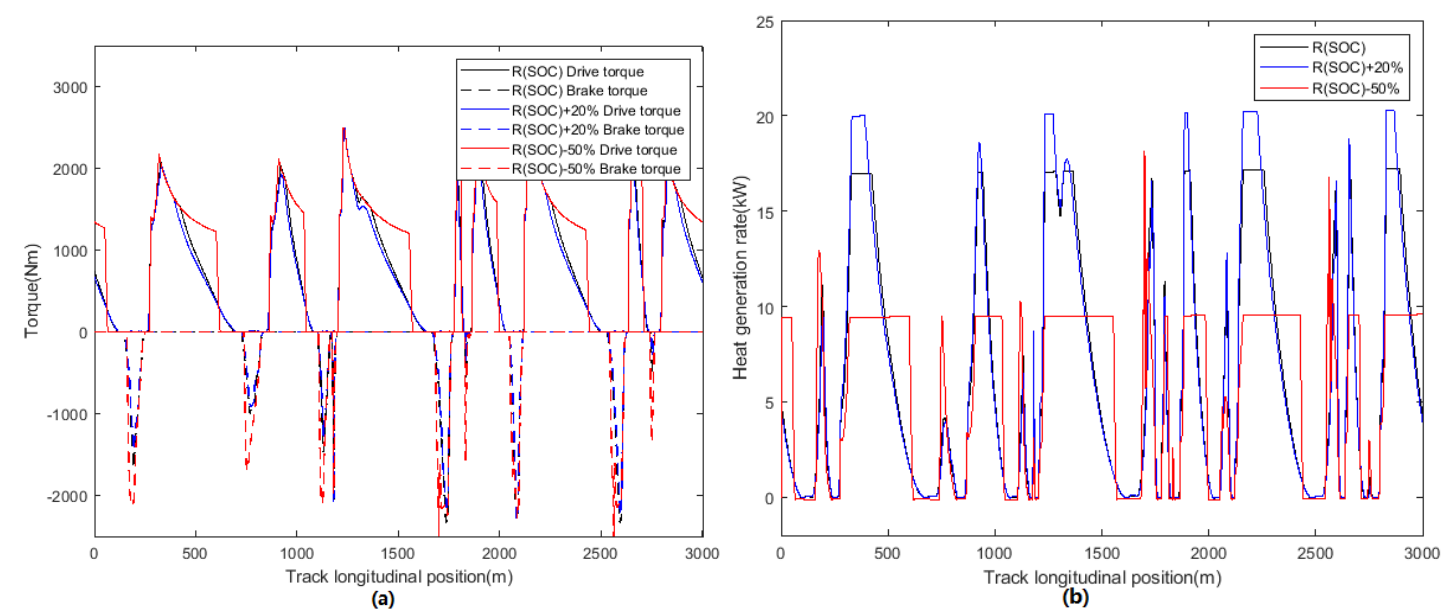

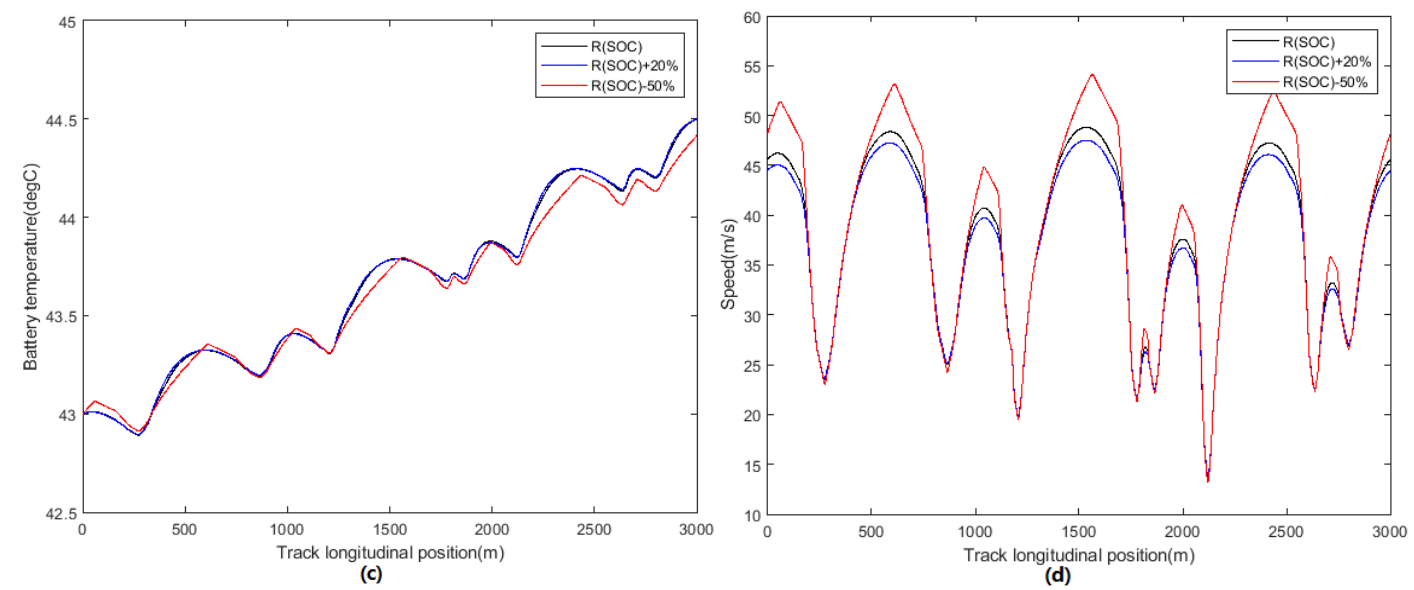

Figure 19: Detailed states and controls under three different cases of battery internal resistance: (a)

Drive and brake torques; (b) heat generation rate; (c) battery temperature; (d) speed profile

It can be observed from figure 19(a) that for Case 3 with 50\% less resistance and not being thermalconstrained, gentle lift-off is not needed. While for the first two cases, Case 2 with higher resistance than Case 1, needs more gentle lift-off and starts earlier like what was observed in section 4.3.2. This leads to a significant lower high-speed performance demonstrated in figure 19(d). It should be noted that in table 6 , only propulsion heat generation peaks are compared. This is because different lift-off strategy leads to different speed at braking point shown by figure 19(b) and (d). With less lift-off, the braking starts at a higher speed thus increases the regeneration power. As a consequence, this makes the peak regeneration power less comparable.

Overall, changes in battery resistance have non-negligible effects on performance, therefore if more detailed data is available, more influence factors should be accounted in the resistance calculation.

\subsection{Comparison between Gen 2 and Gen 3 specs}

Because the details of Gen 3 is yet to be determined by the FIA at the time this study is being completed, the vehicle specs of Gen 3 used here remains the same as that of Gen 2 except that for the formulation of Gen 3 problems, a differential is added to the front axle as stated in Section 2.2.2 and power limit is raised from $200 \mathrm{~kW}$ to $300 \mathrm{~kW}$ in race mode.

Similar to the performance shown in Section 4.3.2, the energy sensitivity of a Gen 3 car follows a similar pattern. The relation between energy per lap and lap time can also be fitted into:

$$
t_{\text {lap }}=3.063 E_{p}^{4}-22.32 E_{p}^{3}+67.24 E_{p}^{2}-100.1 E_{p}+138.3
$$

Comparison between the two shows that with same amount of energy, a Gen 3 car is much faster than the Gen 2 one. Horizontally, with same lap time as target, a Gen 3 car uses $10 \%$ (target of $88 \mathrm{~s}$ ) to $30 \%$ (target of $81 \mathrm{~s}$ ) less energy than a Gen 2 car. The result can be explained in two main aspects. First, as shown in figure 21, with $100 \mathrm{~kW}$ more power going out of the RESS during acceleration, the Gen 3 gains significant speed advantage before braking into a corner when the acceleration is power-limited. It can be seen from a G-G diagram that the Gen 3 car has larger acceleration capacity in the relatively high speed range. Second, as shown in figure 22(a), the Gen 3 car consumes energy at a higher rate (steeper slope) compared to Gen 2. Although the acceleration costs more energy of a Gen 3 car ( 17\% more than Gen 2 as shown figure 22(b)), what makes it remarkably more efficient is the MGU on the front axle which harvests kinetic energy during braking instead of wasting it like 
the Gen 2 through friction brakes. Meanwhile, LaC operation can also be found applicable on Gen3 cars as blocked in figure 22 . The front axle MGU allows a Gen3 car to slightly postpone the start of $\mathrm{LaC}$ to gain more speed at high speeds. By the end of a lap, Gen 3 is able to harvest $55 \%$ more energy in comparison to Gen 2. The cases here illustrated the energy usage of Gen 2 and Gen 3 based on the same energy per lap of $1.6 \mathrm{kWh}$.

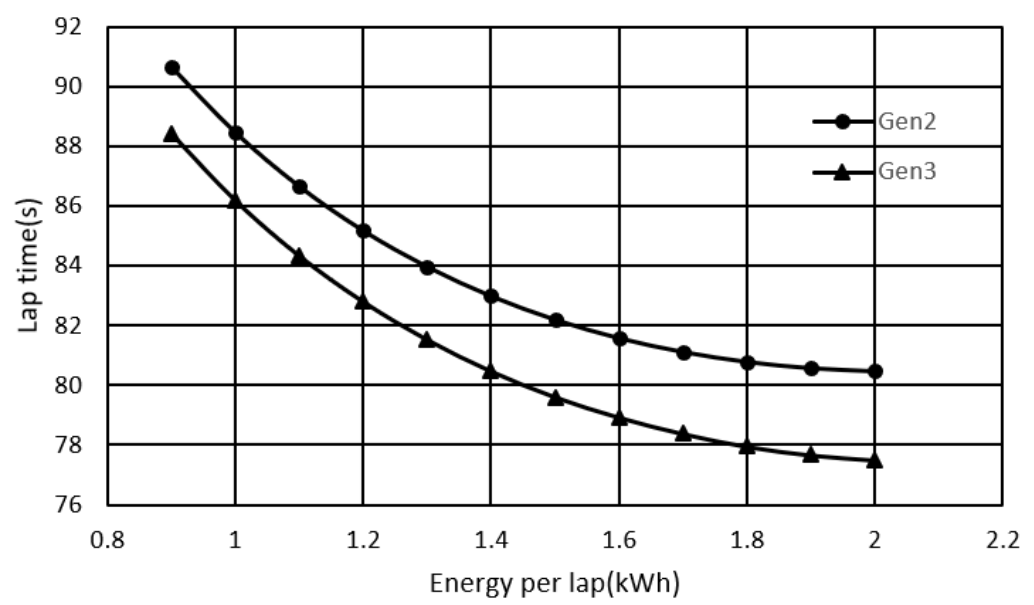

Figure 20: Performance comparison of Gen 2 and Gen 3 Formula-E cars
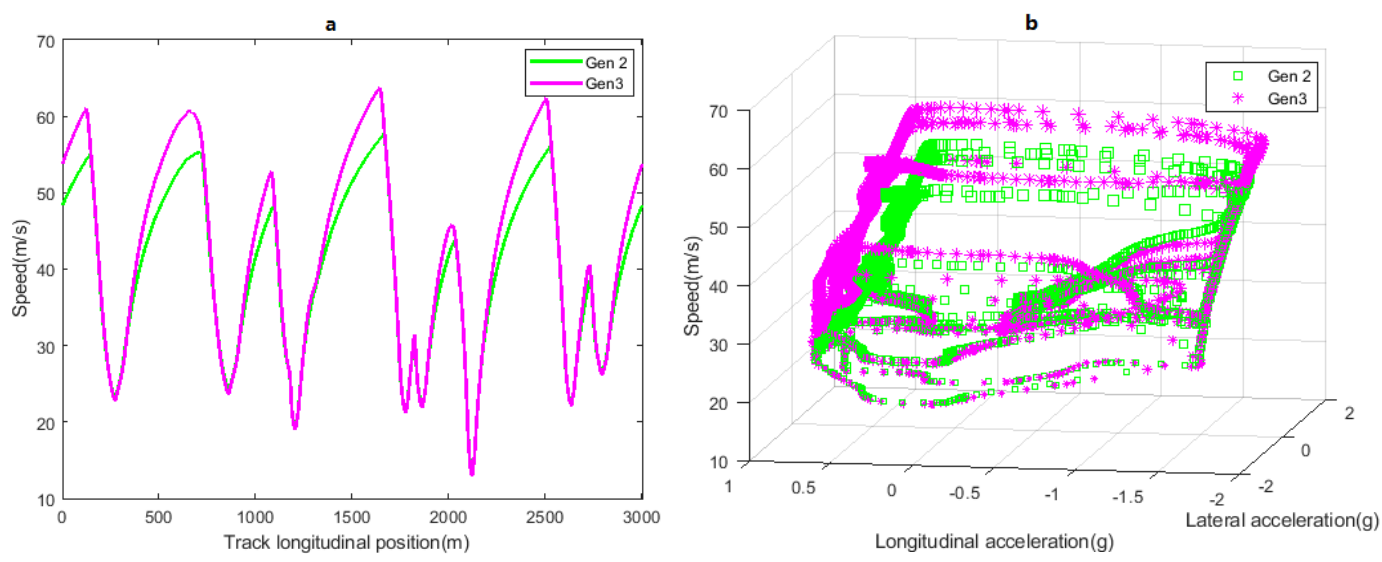

Figure 21: Comparison between Gen 2 and Gen 3: a) speed profile, and b) G-G diagram 

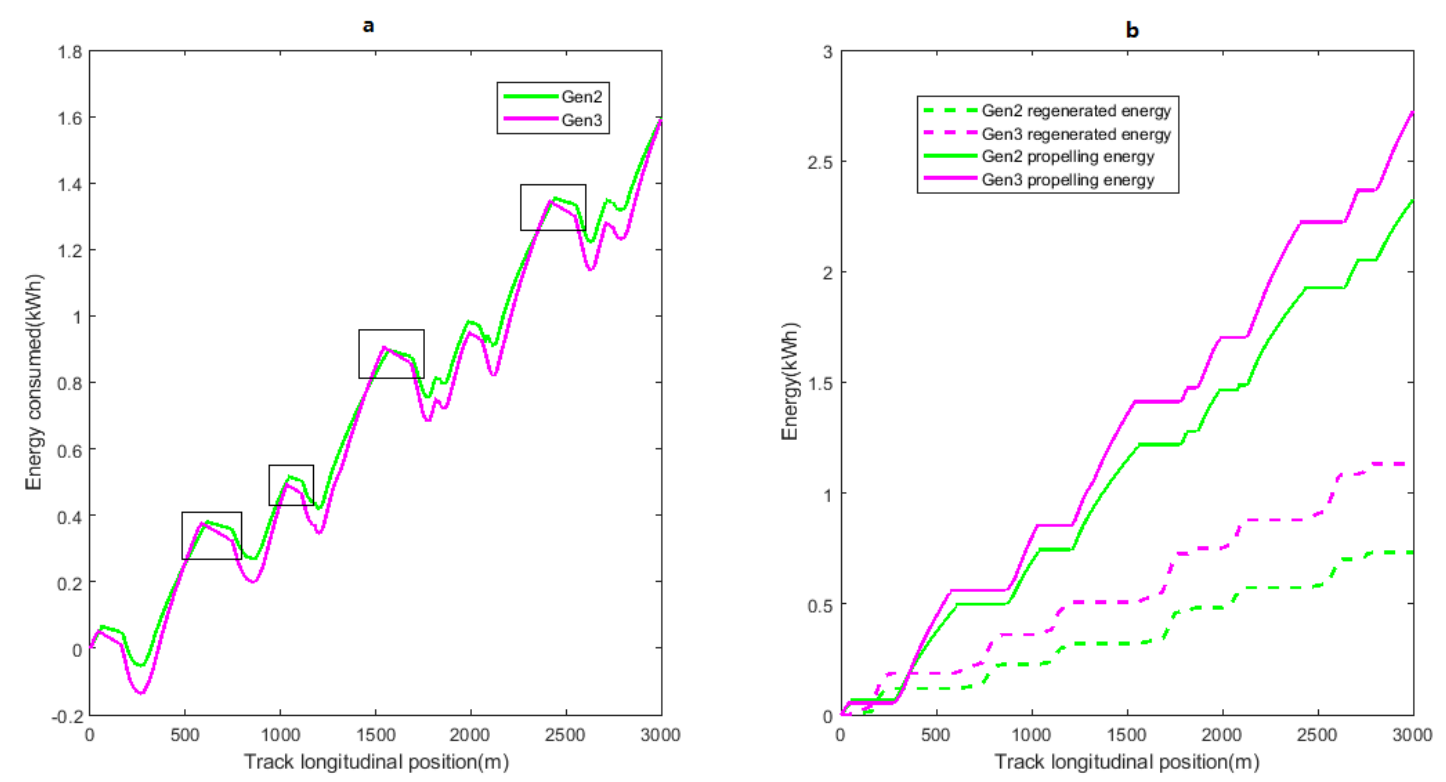

Figure 22: Energy usage comparison between Gen 2 and Gen 3 Formula-E cars
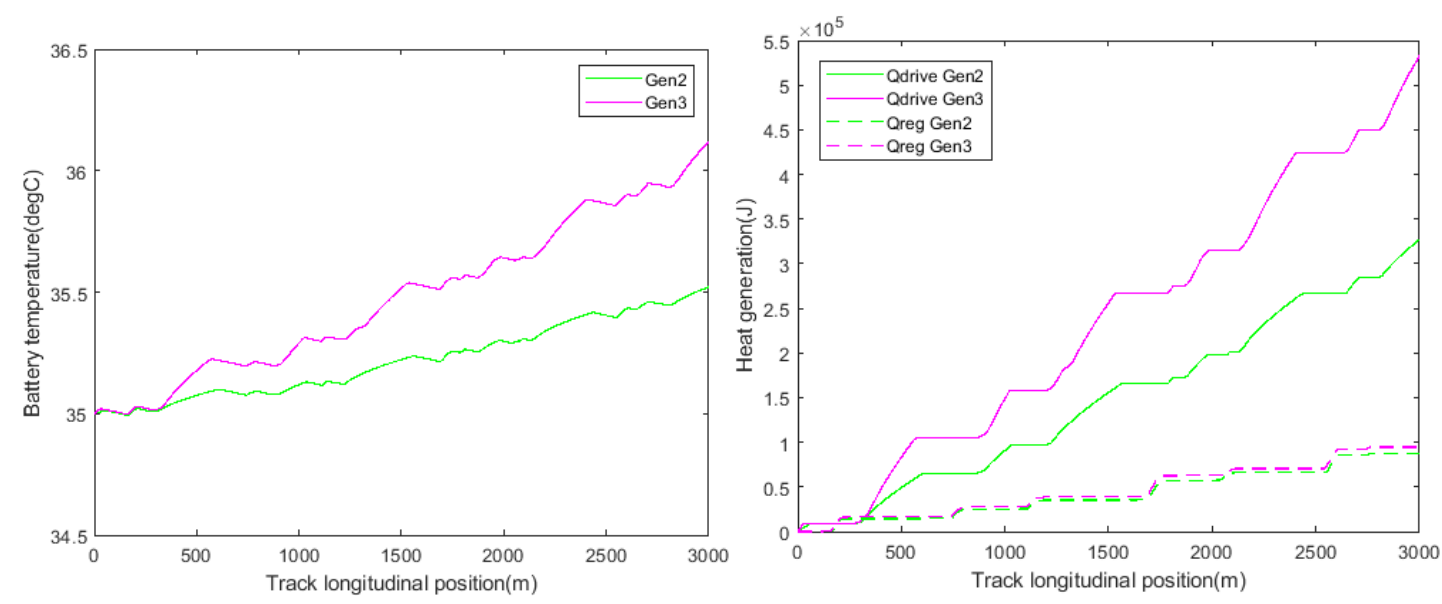

Figure 23: Comparison of thermal behavior in Gen 2 and Gen 3 cars: a) battery temperature b) heat generation component

It is clear that Gen 3 car is more time-energy efficient than Gen 2. In terms of thermal behavior, however, a Gen 3 car would faces more critical situations as shown in figure 23. In that figure, Qdrive denotes the heat generation during propelling and Qreg denotes the heat generated during harvesting.

The examples are given the same initial SOC of $100 \%$ and initial temperature of $35^{\circ} \mathrm{C}$ assuming that the battery and cooling characteristics are the same for Gen 2 and Gen 3 cars. It is observed that Gen 3 suffers from battery heating more than Gen 2 car. The heat generation increases mainly from the propelling phase. It should be noted that theoretically the difference in regeneration part should also be huge. However, the similarity of regeneration heat in this case is due to $k_{r}$ in equation (36) that is adjusted to 0.65 for Gen 3 to comply with the maximum regeneration power stated by the technical regulation. The summary of energy and thermal comparison is presented in Table 7. 
Table 7: Comparison between Gen 2 and Gen 3 simulation models

\begin{tabular}{|l|l|l|l|}
\hline & Gen2 & Gen3 & Difference(compared to Gen2) \\
\hline Energy per lap $(\mathrm{kWh})$ & 1.6 & 1.6 & - \\
\hline Initial SOC & $100 \%$ & $100 \%$ & - \\
\hline Initial Battery temperature $\left({ }^{\circ} \mathrm{C}\right)$ & 35 & 35 & - \\
\hline Propelling energy $(\mathrm{kWh})$ & 2.332 & 2.733 & $+17.2 \%$ \\
\hline Regenerated energy $(\mathrm{kWh})$ & 0.733 & 1.133 & $+54.67 \%$ \\
\hline Lap time $(\mathrm{s})$ & 81.59 & 78.93 & $-3.26 \%$ \\
\hline Temperature rise $\left({ }^{\circ} \mathrm{C}\right)$ & 0.51 & 1.11 & $+117.6 \%$ \\
\hline $\begin{array}{l}\text { Heat generated from propelling } \\
(\mathrm{k} J)\end{array}$ & 324 & 528 & $+63.0 \%$ \\
\hline $\begin{array}{l}\text { Heat generated from regeneration } \\
(\mathrm{kJ})\end{array}$ & 87.3 & 94.6 & $+8.36 \%$ \\
\hline
\end{tabular}

According to FIA, in the future Gen 3 era, fast-charge pitstops might be introduced into the race events. This allows drivers to pit in and perform a fast charging which gives additional $4 \mathrm{kWh}$ energy. This process cost approximately 30 seconds which driver has to make up in the remaining laps. In the case of the Merrakesh track used in this study, a simplified race planning problem without fastcharge pitstop can be formulated as follows:

$$
\begin{gathered}
\operatorname{Min} f=\frac{1}{n} \\
\text { s.t. }\left\{\begin{array}{c}
n E_{p}<51 k W h \\
45 \mathrm{~min}<n t_{\text {lap }}<45 \mathrm{~min}+t_{\text {lap }}
\end{array}\right.
\end{gathered}
$$

where $n$ is the total number of laps, and if pitstop strategy is adopted, the problem can be transferred to:

$$
\begin{gathered}
\operatorname{Min} f=\frac{1}{n_{1}+n_{2}} \\
\text { s.t. }\left\{\begin{array}{c}
n_{1} E_{p 1}+n_{2} E_{p 2}<51 k W h+4 k W h \\
45 \min <n_{1} t_{\text {lap } 1}+n_{2} t_{\text {lap } 2}+30 s<45 \mathrm{~min}+t_{\text {lap } 2}
\end{array}\right.
\end{gathered}
$$

The subscript 1 and 2 denotes the value before and after the fast-charge pitstop respectively. For the non-pitstop problem, the solution is $n=34$ and lap time of $79.62 \mathrm{~s}$, while for the fast-charge strategy, the solution is also $n_{1}+n_{2}=34$ and with slight improvement of lap time to $78.82 \mathrm{~s}$. However, the cost of 30 seconds of time is too much that it turns out the total race time with fastcharge pitstop is $2.8 \mathrm{~s}$ longer which suggests that the fast-charging is not favorable in this simplified case at Merrakesh track. It could be potentially favorable if the charging time can be reduced by such $2.8 \mathrm{~s}$ or perhaps on another track. And it should also be noted that such fast charging might generate huge amount of heat which might ruin the thermal management. Therefore the worthiness of a fast-charging pitstop is still under investigation. This is not the main concern of this study therefore is not further discussed.

\section{Conclusions}

The management of energy and thermal behavior of a Formula-E car battery was studied as one of the most crucial parts of formula-E race strategies. Optimal control techniques were applied to 
obtain optimal control signals of steering, acceleration, braking, and regeneration under different energy and thermal restrictions. A battery thermal model was used to give an insight into the details of heat generation during a race. The results demonstrated how the control inputs should be adapted to various restrictions in real-time. The lift and coasting technique was investigated as the most time-efficient way to manage the energy. For thermal constraints, smooth drive torque decrease was concluded to be the most effective way for reducing battery temperature rise. Finally, a perspective study of the future Gen 3 formula-E car was presented and the simulation results were compared to the current Gen 2 car. It was concluded that whilst Gen 3 is more energy-efficient ( $10 \%$ to $30 \%$ less energy consumption) by having energy harvest from the front axle, the cooling system need to be more powerful to overcome the potential heating risk $(60 \%$ more heat generation).

For the first time, in this study, 1) the effect of combined energy and thermal constraints to lap time has been studied; 2) the impact of introducing front axle energy harvesting on performance was investigated; 3 ) a novel effective technique to reduce temperature rise has been found

\section{References}

[1] Song, Ziyou, et al. "Energy management strategies comparison for electric vehicles with hybrid energy storage system." Applied Energy 134 (2014): 321-331.

[2] Hu, Xiaosong, et al. "Optimal energy management and sizing of a dual motor-driven electric powertrain." IEEE Transactions on Power Electronics 34.8 (2018): 7489-7501.

[3] Song, Chuanxue, et al. "Energy management of parallel-connected cells in electric vehicles based on fuzzy logic control." Energies 10.3 (2017): 404.

[4] Hou, Cong, et al. "Approximate Pontryagin's minimum principle applied to the energy management of plug-in hybrid electric vehicles." Applied Energy 115 (2014): 174-189.

[5] Chen, Zheng, et al. "A novel energy management method for series plug-in hybrid electric vehicles." Applied Energy 145 (2015): 172-179.

[6] Gong, Qiuming, Yaoyu Li, and Zhong-Ren Peng. "Trip-based optimal power management of plug-in hybrid electric vehicles." IEEE Transactions on vehicular technology 57.6 (2008): 33933401.

[7] de l'Automobile, Fédération Internationale. "2020 FIA Formula One Championship Technical Regulations and Sporting Regulations." Paris: Fédération Internationale de l'Automobile (2019).

[8] de 1'Automobile, Fédération Internationale. "2020 FIA Formula E Championship Technical Regulations and Sporting Regulations." Paris: Fédération Internationale de l'Automobile (2019).

[9] Cammaerts, Kia, Phil Morse, and Kazuharu Kidera. "Improving Performance through the Use of Driver-in-the-Loop Simulations." ATZ worldwide 121.1 (2019): 52-57.

[10] Brayshaw, D. L., and M. F. Harrison. "A quasi steady state approach to race car lap simulation in order to understand the effects of racing line and centre of gravity location." Proceedings of the Institution of Mechanical Engineers, Part D: Journal of Automobile Engineering 219.6 (2005): 725 739.

[11] Wieczorek, Maciej, and Mirosław Lewandowski. "A mathematical representation of an energy management strategy for hybrid energy storage system in electric vehicle and real time optimization using a genetic algorithm." Applied energy 192 (2017): 222-233.

[12] Cossalter, Vittore, et al. "A general method for the evaluation of vehicle manoeuvrability with special emphasis on motorcycles." Vehicle system dynamics 31.2 (1999): 113-135.

[13] Tavernini, Davide, et al. "Minimum time cornering: the effect of road surface and car 
transmission layout." Vehicle System Dynamics 51.10 (2013): 1533-1547.

[14] Pontryagin, Lev Semenovich. Mathematical theory of optimal processes. Routledge, 2018.

[15] Wächter, Andreas, and Lorenz T. Biegler. "On the implementation of an interior-point filter line-search algorithm for large-scale nonlinear programming." Mathematical programming 106.1 (2006): 25-57.

[16] Casanova D. On minimum time vehicle manoeuvring: the theoretical optimal lap [PhD thesis]. Bedford:Cranfield University, School of Engineering; 2000

[17] Dal Bianco, Nicola, et al. "Comparison of direct and indirect methods for minimum lap time optimal control problems." Vehicle System Dynamics 57.5 (2019): 665-696.

[18] Perantoni, Giacomo, and David JN Limebeer. "Optimal control for a formula one car with variable parameters." Vehicle System Dynamics 52.5 (2014): 653-678.

[19] Perantoni, Giacomo, and David JN Limebeer. "Optimal control of a formula one car on a threedimensional track-Part 1: Track modeling and identification." Journal of Dynamic Systems, Measurement, and Control 137.5 (2015).

[20] Tremlett, Anthony J., et al. "Optimal control of motorsport differentials." Vehicle System Dynamics 53.12 (2015): 1772-1794.

[21] Kelly, Daniel Patrick, and Robin S. Sharp. "Time-optimal control of the race car: influence of a thermodynamic tyre model." Vehicle system dynamics 50.4 (2012): 641-662.

[22] Tremlett, A. J., and D. J. N. Limebeer. "Optimal tyre usage for a Formula One car." Vehicle System Dynamics 54.10 (2016): 1448-1473.

[23] Limebeer, David JN, Giacomo Perantoni, and Anil V. Rao. "Optimal control of formula one car energy recovery systems." International Journal of Control 87.10 (2014): 2065-2080.

[24] Masouleh, Mehdi Imani, and David JN Limebeer. "Optimizing the aero-suspension interactions in a formula one car." IEEE Transactions on Control Systems Technology 24.3 (2015): 912-927.

[25] Benson, David A., et al. "Direct trajectory optimization and costate estimation via an orthogonal collocation method." Journal of Guidance, Control, and Dynamics 29.6 (2006): 1435-1440.

[26] Jonathan M. Gitlin - Jan 30, 2018 4:36 pm UTC. "Formula E's New Electric Car Looks like Nothing Else in Racing." Ars Technica, 30 Jan. 2018, arstechnica.com/cars/2018/01/formula-esnew-electric-car-looks-like-nothing-else-in-racing/.

[27] Mitchell, Scott. "Why Formula E Is so Hard - Autosport Engineering." Autosport.com, www.autosport.com/engineering/feature/8211/why-formula-e-is-so-hard.

[28] Pacejka, Hans. Tire and vehicle dynamics. Elsevier, 2005.

[29] Kelly DP. Lap time simulation with transient vehicle and tyre dynamics [ $\mathrm{PhD}$ thesis]. Bedford: Cranfield University, School of Engineering; 2008.

[30] Abada, Sara, et al. "Examination and modeling of thermal runaway issues pertaining to new and aged Li-ion batteries." 2015.

[31] Abada, Sara, et al. "Safety focused modeling of lithium-ion batteries: A review." Journal of Power Sources 306 (2016): 178-192.

[32] Onda, Kazuo, et al. "Thermal behavior of small lithium-ion battery during rapid charge and discharge cycles." Journal of Power sources 158.1 (2006): 535-542.

[33] Bernardi, D., E. Pawlikowski, and John Newman. "A general energy balance for battery systems." Journal of the electrochemical society 132.1 (1985): 5-12.

[34] Al Hallaj, Said, J. Prakash, and J. R. Selman. "Characterization of commercial Li-ion batteries using electrochemical-calorimetric measurements." Journal of Power Sources 87.1-2 (2000): 186- 
194.

[35]Gu, W. B., and C. Y. Wang. "Thermal - electrochemical modeling of battery systems." Journal of The Electrochemical Society 147.8 (2000): 2910-2922.

[36] Chen, S. C., C. C. Wan, and Y. Y. Wang. "Thermal analysis of lithium-ion batteries." Journal of power sources 140.1 (2005): 111-124.

[37] Liu, Xuze, and Abbas Fotouhi. "Formula-E race strategy development using artificial neural networks and Monte Carlo Tree Search." (2020).

[38] Masouleh, Mehdi Imani. Optimal control and stability of four-wheeled vehicles. Diss. University of Oxford, 2017.

[39] Schwartz, Adam Lowell. Theory and implementation of numerical methods based on RungeKutta integration for solving optimal control problems. Diss. University of California, Berkeley, 1996.

[40] Davis, Philip J., and Philip Rabinowitz. Methods of numerical integration. Courier Corporation, 2007.

[41] Patterson, Michael A., and Anil V. Rao. "GPOPS-II: A MATLAB software for solving multiplephase optimal control problems using hp-adaptive Gaussian quadrature collocation methods and sparse nonlinear programming." ACM Transactions on Mathematical Software (TOMS) 41.1 (2014): 1-37..

[42] Wächter, Andreas, and Lorenz T. Biegler. "On the implementation of an interior-point filter line-search algorithm for large-scale nonlinear programming." Mathematical programming 106.1 (2006): 25-57.

[43] Patterson, Michael A., William W. Hager, and Anil V. Rao. "A ph mesh refinement method for optimal control." Optimal Control Applications and Methods 36.4 (2015): 398-421.

[44] Betts, John T. Practical methods for optimal control and estimation using nonlinear programming. Vol. 19. Siam, 2010.

[45] Waag, Wladislaw, Stefan Käbitz, and Dirk Uwe Sauer. "Experimental investigation of the lithium-ion battery impedance characteristic at various conditions and aging states and its influence on the application." Applied energy 102 (2013): 885-897.

[46] Broussely, Michel, et al. "Main aging mechanisms in Li ion batteries." Journal of power sources 146.1-2 (2005): 90-96.

\section{Appendix}
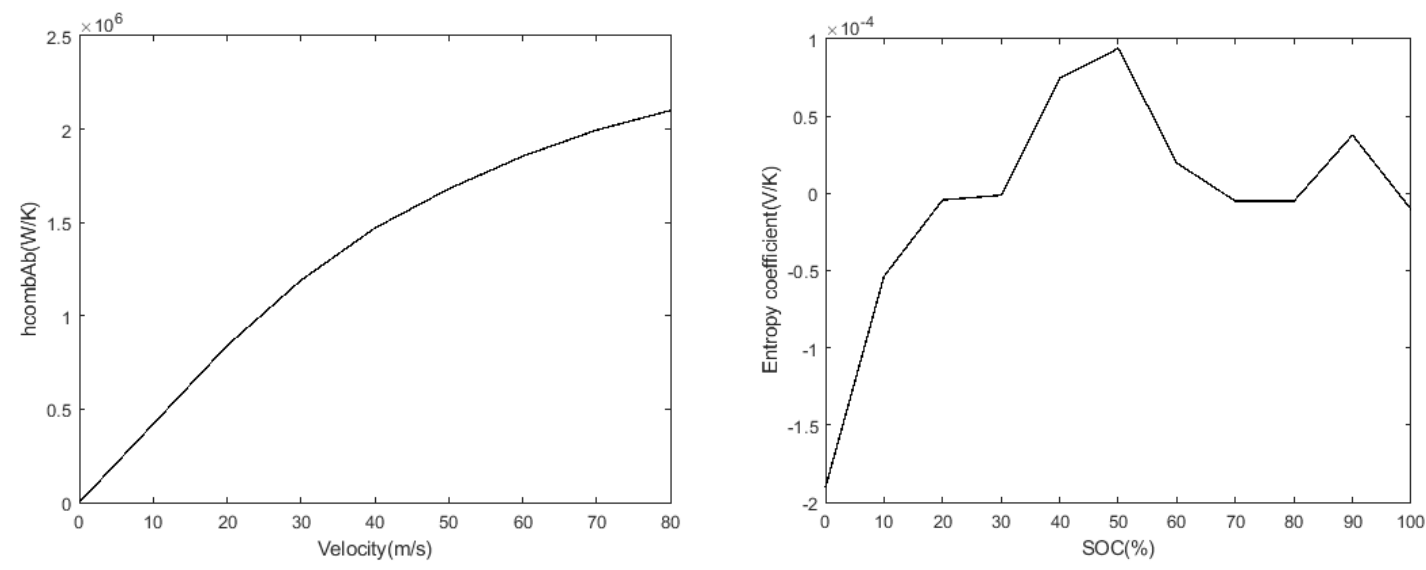
Figure A1

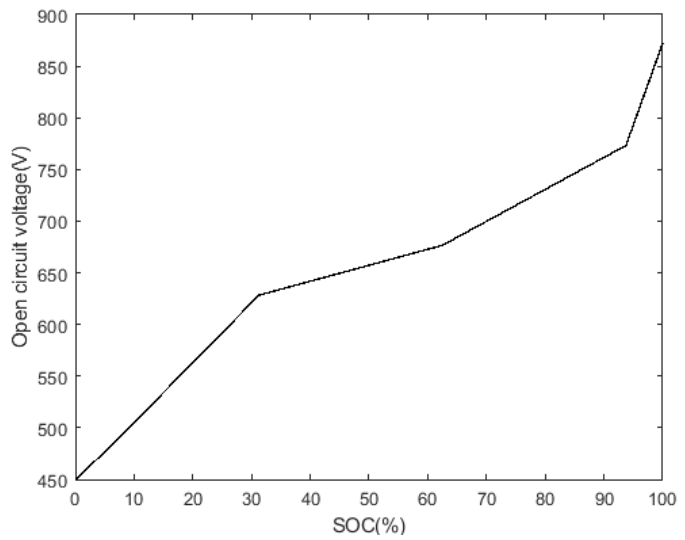

Figure A3
Figure A2

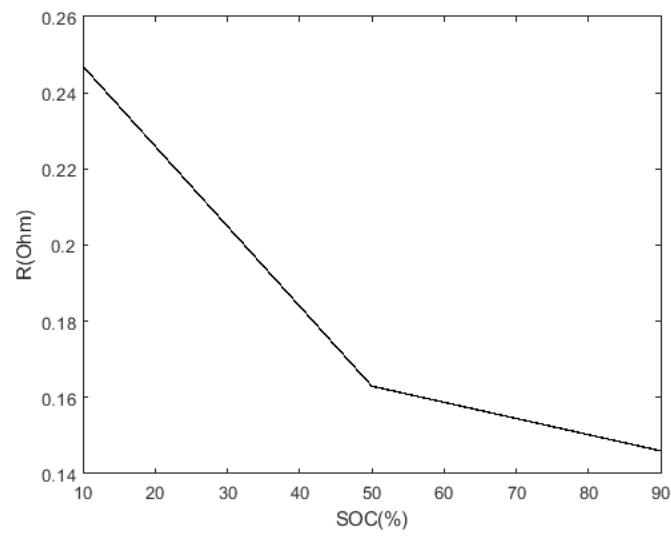

Figure A4

Figure A1 is the 'Combined heat transfer coefficient $h_{c o m b}(V) A_{b}$ ' appeared in equation 50

Figure A2 is the 'Entropy coefficient $\left(\frac{d U_{o c_{b}}}{d T_{b}}\right)(S O C)$ ' appeared in equation46

Figure A3 is the 'Open circuit voltage $U_{\text {oc_b }}$ ' profile of the battery package in equation 44

Figure A4 is the 'Combined resistance $R(S O C)$ ' appeared in equation 46

The table below shows other information in the model for result reproduction

Table A1

\begin{tabular}{|l|l|}
\hline Parameters & Value \\
\hline Battery cell number & 209 \\
\hline Battery mass & $317 \mathrm{~kg}$ \\
\hline Battery specific heat capacity & $1015 \mathrm{~J} /(\mathrm{kg} \cdot \mathrm{K})$ \\
\hline Coolant mass & $20 \mathrm{~kg}$ \\
\hline Coolant specific heat capacity & $1800 \mathrm{~J} /(\mathrm{kg} \cdot \mathrm{K})$ \\
\hline
\end{tabular}


2020-09-13

\title{
Optimal energy management for formula-E cars with regulatory limits and thermal constraints
}

\author{
Liu, Xuze
}

Elsevier

Liu X, Fotouhi A, Auger DJ. (2020) Optimal energy management for formula-E cars with regulatory limits and thermal constraints. Applied Energy, Volume 279, December 2020, Article number 115805

https://doi.org/10.1016/j.apenergy.2020.115805

Downloaded from Cranfield Library Services E-Repository 